\title{
CLOSED CONVEX HULLS OF UNITARY ORBITS IN VON NEUMANN ALGEBRAS
}

\author{
FUMIO HIAI AND YOSHIHIRO NAKAMURA
}

\begin{abstract}
Let $\mathscr{M}$ be a von Neumann algebra. The distance $\operatorname{dist}(x, \operatorname{co} \mathscr{U}(y))$ between $x$ and $\operatorname{co} \mathscr{U}(y)$ for selfadjoint operators $x, y \in \mathscr{M}$ and the distance $\operatorname{dist}(\varphi, \operatorname{co} \mathscr{U}(\psi))$ between $\varphi$ and $\operatorname{co} \mathscr{U}(\psi)$ for selfadjoint elements $\varphi, \psi \epsilon$ $\mathscr{M}_{*}$ are exactly estimated, where co $\mathscr{U}(y)$ and co $\mathscr{U}(\psi)$ are the convex hulls of the unitary orbits of $y$ and $\psi$, respectively. This is done separately in the finite factor case, in the infinite semifinite factor case, and in the type III factor case. Simple formulas of distances between two convex hulls of unitary orbits are also given. When $\mathscr{M}$ is a von Neumann algebra on a separable Hilbert space, the above cases altogether are combined under the direct integral decomposition of $\mathscr{M}$ into factors. As a result, it is known that if $\mathscr{M}$ is $\sigma$-finite and $x \in \mathscr{M}$ is selfadjoint, then $\overline{\mathrm{co}} \mathscr{U}(x)=\overline{\mathrm{co}}^{\mathrm{w}} \mathscr{U}(x)$ where $\overline{\mathrm{co}} \mathscr{U}(x)$ and $\overline{\mathrm{co}}^{\mathrm{w}} \mathscr{U}(x)$ are the closures of $\operatorname{co} \mathscr{U}(x)$ in norm and in the weak operator topology, respectively.
\end{abstract}

\section{INTRODUCTION}

Let $\mathscr{M}$ be a von Neumann algebra and $\mathscr{M}_{\text {sa }}$ (resp. $\mathscr{M}_{*, \text { sa }}$ ) be the set of all selfadjoint elements in $\mathscr{M}$ (resp. in the predual $\mathscr{M}_{*}$ ). For $x \in \mathscr{M}$, let $\operatorname{co} \mathscr{U}(x)$ denote the convex hull of the unitary orbit $\mathscr{U}(x)=\left\{u x u^{*}: u \in \mathscr{M}\right.$ unitary $\}$. Let $\overline{\operatorname{co}} \mathscr{U}(x)$ and $\overline{\operatorname{co}}^{\mathrm{w}} \mathscr{U}(x)$ be the closures of co $\mathscr{U}(x)$ in norm and in the weak operator topology, respectively. It is very important in theory of von Neumann algebras to consider these closed convex hulls of unitary orbits of operators. For instance, several authors discussed the intersection of $\overline{\mathrm{co}} \mathscr{U}(x)$ or $\overline{\mathrm{co}}^{\mathrm{w}} \mathscr{U}(x)$ with the center $\mathscr{Z}$ of $\mathscr{M}$ (see e.g. [9, 10, 17, 34, 37]). According to [10] (or $[12, \S$ III.5]), any von Neumann algebra $\mathscr{M}$ has the Dixmier property asserting nonemptiness of $\overline{\mathrm{co}} \mathscr{U}(x) \cap \mathscr{Z}$ for every $x \in \mathscr{M}$, and in particular if $\mathscr{M}$ is finite, then $\overline{\mathrm{co}} \mathscr{U}(x) \cap \mathscr{Z}=\overline{\mathrm{co}}^{\mathrm{w}} \mathscr{U}(x) \cap \mathscr{Z}=\left\{x^{\natural}\right\}$ for every $x \in \mathscr{M}$ where $x \mapsto x^{\natural}$ is the center-valued trace on $\mathscr{M}$. When $\mathscr{M}$ is properly infinite, $\overline{c o} \mathscr{U}(x) \cap \mathscr{Z}$ and $\overline{c o}^{\mathrm{w}} \mathscr{U}(x) \cap \mathscr{Z}$ are regarded as certain types of essential spectrum or essential numerical range of $x[9,17]$. It was shown in [17] that if $\mathscr{M}$ is $\sigma$-finite, then $\overline{\operatorname{co}} \mathscr{U}(x) \cap \mathscr{Z}=\overline{\mathrm{co}}^{\mathrm{w}} \mathscr{U}(x) \cap \mathscr{Z}$ for all $x \in \mathscr{M}$. Indeed the last assertion holds if and only if $\mathscr{M}$ satisfies a condition slightly weaker than $\sigma$-finiteness [34]. However it seems that there is no literature dealing with the direct relation between $\overline{\operatorname{co}} \mathscr{U}(x)$ and $\overline{\mathrm{co}}^{\mathrm{w}} \mathscr{U}(x)$.

Received by the editors June 16, 1988 and, in revised form, January 23, 1989.

1980 Mathematics Subject Classification (1985 Revision). Primary 46L10, 46L50.

Research supported in part by Grant-in-Aid for Scientific Research. 
The study of majorization was developed for real-valued measurable functions based on theory of rearrangements (see e.g. [5, 35]). Moreover the majorization theory plays a vital role in the study of matrices via the comparison of eigenvalues or singular values of matrices [2, 26, 27, 40]. The noncommutative integration and probability theory (in the semifinite case) was founded in $[11,31,36,41]$. The concept of $\tau$-measurable operators was introduced in [30], which gives a nice foundation for noncommutative $L^{p}$-spaces $L^{p}(\mathscr{M})$ on semifinite von Neumann algebras $\mathscr{M}$. The majorization theory in semifinite von Neumann algebras was recently developed in [18-20, 23, 24, 28] by using the notion of generalized $s$-numbers or spectral scales of $\tau$-measurable operators. A most recent and general exposition on generalized $s$-numbers of $\tau$-measurable operators is found in [13]. It is known [18, 19, 23, 24] that the condition of unitary mixing is closely connected with that of majorization. For instance, when $\mathscr{M}$ is a semifinite factor, if $x$ and $y$ are positive operators in $L^{1}(\mathscr{M})$, then $x$ is in the $L^{1}$-closure of $\operatorname{co} \mathscr{U}(y)$ if and only if $x$ is majorized by $y$ [18]. Theory of unitary mixing in the state spaces of operator algebras was considerably studied in [1] in more general setup. Using the majorization technique, we obtained in [21] the formula of $L^{p}$-distance between unitary orbits of $\tau$-measurable selfadjoint operators in terms of their spectral scales. Furthermore the majorization theory can be applied to the study of $L^{p}$ distance of unitary orbits in Haagerup $L^{p}$-spaces [15] even when $\mathscr{M}$ is a type III factor. See [21] in case of Haagerup $L^{p}$-spaces associated with continuous decompositions of type III $_{1}$ factors, and [29] in case of those associated with discrete decompositions of type III $_{\lambda}$ factors, $0<\lambda<1$.

The main aim of this paper is to estimate the distance $\operatorname{dist}(x, \operatorname{co} \mathscr{U}(y))$ between $x$ and $\operatorname{co} \mathscr{U}(y)$ for $x, y \in \mathscr{M}_{\text {sa }}$ and the distance $\operatorname{dist}(\varphi, \operatorname{co} \mathscr{U}(\psi))$ for $\varphi, \psi \in \mathscr{M}_{* \text {, sa }}$ where co $\mathscr{U}(\psi)$ denotes the convex hull of the unitary orbit $\mathscr{U}(\psi)=\left\{\psi\left(u^{*} \cdot u\right): u \in \mathscr{M}\right.$ unitary $\}$. After giving notations and preliminaries on majorization in $\S 1$, we divide this study into the finite factor case $(\S 2)$, the infinite semifinite factor case $(\S 3)$, and the type III factor case $(\S 4)$. In each of the above cases, before estimating $\operatorname{dist}(x, \operatorname{co} \mathscr{U}(y))$ for $x, y \in \mathscr{M}_{\mathrm{sa}}$, we characterize a special condition of $x$ being in $\overline{c o} \mathscr{U}(y)$. In $\S \S 2$ and 3 , we obtain the formulas of $\operatorname{dist}(x, \operatorname{co} \mathscr{U}(y))$ for $x, y \in \mathscr{M}_{\text {sa }}$ and of the $L^{1}$ distance $\operatorname{dist}_{L^{1}}(x, \operatorname{co} \mathscr{U}(y))$ for $x, y \in L^{1}(\mathscr{M})_{\text {sa }}\left(=\mathscr{M}_{* \text {, sa }}\right)$ in terms of the spectral scales of $x$ and $y$. Here the majorization technique is useful. In $\S 4$, $\operatorname{dist}(x, \operatorname{co} \mathscr{U}(y))$ for normal operators $x, y \in \mathscr{M}$ is estimated in terms of the spectra of $x$ and $y$. Also $\operatorname{dist}(\varphi, \operatorname{co} \mathscr{U}(\psi))$ for $\varphi, \psi \in \mathscr{M}_{* \text {, sa }}$ is simply estimated in terms of $\|\varphi\|,\|\psi\|$ and $\varphi(1), \psi(1)$. Furthermore, when $\mathscr{M}$ is a type III $_{1}$ factor, the formula of the $L^{p}$-distance $\operatorname{dist}_{L^{p}}(x$, co $\mathscr{U}(y))$ is obtained for selfadjoint elements $x, y$ in the Haagerup $L^{p}$-space. In $\S 5$, we give some simple formulas of the distance $\operatorname{dist}(\operatorname{co} \mathscr{U}(x), \operatorname{co} \mathscr{U}(y))$ between co $\mathscr{U}(x)$ and $\operatorname{co} \mathscr{U}(y)$ for $x, y \in \mathscr{M}_{\text {sa }}$ and of the distance $\operatorname{dist}(\operatorname{co} \mathscr{U}(\varphi), \operatorname{co} \mathscr{U}(\psi))$ for $\varphi$, $\psi \in \mathscr{M}_{* \text {, sa }}$. Finally in $\S 6$, when $\mathscr{M}$ is a von Neumann algebra on a separable 
Hilbert space, we give the estimates of $\operatorname{dist}(x, \operatorname{co} \mathscr{U}(y))$ for $x, y \in \mathscr{M}_{\text {sa }}$ and of $\operatorname{dist}(\varphi, \operatorname{co} \mathscr{U}(\psi))$ for $\varphi, \psi \in \mathscr{M}_{* \text {,sa }}$ under the direct integral decomposition of $\mathscr{M}$ into factors. But the proofs are only sketched and the details are left to the reader. Furthermore we know an important result that $\overline{\mathrm{co}} \mathscr{U}(x)=\overline{\mathrm{co}}^{\mathrm{w}} \mathscr{U}(x)$ holds for every $x \in \mathscr{M}_{\text {sa }}$ whenever $\mathscr{M}$ is $\sigma$-finite.

\section{Preliminaries on spectral scales and majorization}

In this section, we collect notations, definitions and preliminary results. Let $\mathscr{M}$ be an arbitrary von Neumann algebra and $\mathscr{U}$ be the set of all unitaries in $\mathscr{M}$. For each $y \in \mathscr{M}$, let $\mathscr{U}(y)$ denote the unitary orbit $\left\{u y u^{*}: u \in \mathscr{U}\right\}$ of $y$, co $\mathscr{U}(y)$ the convex hull of $\mathscr{U}(y), \overline{c o} \mathscr{U}(y)$ the $\|\cdot\|$-closure of co $\mathscr{U}(y)$, and $\overline{\mathrm{co}}^{\mathrm{w}} \mathscr{U}(y)$ the closure of $\operatorname{co} \mathscr{U}(y)$ in the weak operator topology. For $x, y \in \mathscr{M}$, the $\|\cdot\|$-distance between $x$ and $\operatorname{co} \mathscr{U}(y)$ is written by $\operatorname{dist}(x, \operatorname{co} \mathscr{U}(y))$. Given $\psi \in \mathscr{M}_{*}$, let co $\mathscr{U}(\psi)$ denote the convex hull of $\mathscr{U}(\psi)=\left\{u \psi u^{*}: u \in \mathscr{U}\right\}$ where $a \psi a^{*}=\psi\left(a^{*} \cdot a\right)$ for $a \in \mathscr{M}, \overline{\mathrm{co}} \mathscr{U}(\psi)$ the closure of $\operatorname{co} \mathscr{U}(\psi)$ in norm, and $\operatorname{dist}(\varphi, \operatorname{co} \mathscr{U}(\psi))$ the distance between $\varphi \in \mathscr{M}_{*}$ and $\operatorname{co} \mathscr{U}(\psi)$. When $e \in \mathscr{M}$ is a projection, we denote by $\mathscr{U}_{e}$ the set of all unitaries in $\mathscr{M}_{e}=e \mathscr{M} e$. Also let $\mathscr{U}_{e}(y)=\left\{u y u^{*}: u \in \mathscr{U}_{e}\right\}$ for $y \in \mathscr{M}_{e}$ and $\mathscr{U}_{e}(\psi)=\left\{u \psi u^{*}: u \in \mathscr{U}_{e}\right\}$ for $\psi \in\left(\mathscr{M}_{e}\right)_{*}$.

In the sequel of this section, let $\mathscr{M}$ be a semifinite von Neumann algebra on a Hilbert space $\mathscr{H}$ with a faithful normal semifinite trace $\tau$. A densely defined closed operator $x$ affiliated with $\mathscr{M}$ is said to be $\tau$-measurable if there is, given $\delta>0$, a projection $e \in \mathscr{M}$ such that $e \mathscr{H} \subseteq \mathscr{D}(x)$ and $\tau\left(e^{\perp}\right)<\delta$ where $e^{\perp}=1-e$. We denote by $\widetilde{\mathscr{M}}$ the set of all $\tau$-measurable operators affiliated with $\mathscr{M}$. Then $\widetilde{\mathscr{M}}$ is a complete Hausdorff topological $*$-algebra in the measure topology [30, 39]. For a subspace $\mathscr{L}$ of $\widetilde{\mathscr{M}}$, the set of all selfadjoint (resp. positive selfadjoint) operators in $\mathscr{L}$ is denoted by $\mathscr{L}_{\text {sa }}$ (resp. $\mathscr{L}_{+}$). For each $x \in \widetilde{\mathscr{M}}_{\mathrm{sa}}$, let $e_{E}(x)$ denote the spectral projection of $x$ corresponding to a Borel set $E$ in $\mathbf{R}$. For $1 \leq p<\infty$, the noncommutative $L^{p}$-space $L^{p}(\mathscr{M})$ on $(\mathscr{M}, \tau)$ is the Banach space consisting of all $x \in \widetilde{\mathscr{M}}$ such that the norm $\|x\|_{p}=\tau\left(|x|^{p}\right)^{1 / p}$ is finite (see [11,30,36, 42]). Moreover let $\widetilde{\mathfrak{S}}$ be the set of all $x \in \widetilde{\mathscr{M}}$ such that $\tau\left(e_{(s, \infty)}(|x|)\right)<\infty$ for any $s>0$, and let $\mathfrak{S}=\widetilde{\mathfrak{S}} \cap \mathscr{M}$. For $1 \leq p<\infty, L^{p}(\mathscr{M})$ is included in $\widetilde{\mathfrak{S}}$. In particular when $\mathscr{M}=\mathbf{B}(\mathscr{H})$ the algebra of all bounded operators on $\mathscr{H}, L^{p}(\mathscr{M})$ is the Schatten-von Neumann $p$-class and $\mathfrak{S}=\widetilde{\mathfrak{S}}$ is the algebra of all compact operators on $\mathscr{H}$.

For each $y \in \widetilde{\mathscr{M}}$, let co $\mathscr{U}(y)$ denote the convex hull of $\mathscr{U}(y)=\left\{u y u^{*}: u \in\right.$ $\mathscr{U}\}$ in $\widetilde{\mathscr{M}}$ and $\overline{\mathrm{co}}^{m} \mathscr{U}(y)$ its closure in the measure topology. When $x, y \in$ $L^{p}(\mathscr{M}), 1 \leq p<\infty$, the $\|\cdot\|_{p}$-closure of co $\mathscr{U}(y)$ and the $\|\cdot\|_{p}$-distance between $x$ and co $\mathscr{U}(y)$ are written by $\overline{c o}^{L^{p}} \mathscr{U}(y)$ and $\operatorname{dist}_{L^{p}}(x, \operatorname{co} \mathscr{U}(y))$, respectively.

The generalized s-number $\mu_{t}(x), t>0$, of $x \in \widetilde{\mathscr{M}}$ is defined by

$$
\mu_{t}(x)=\inf \left\{s \geq 0: \tau\left(e_{(s, \infty)}(|x|)\right) \leq t\right\} .
$$


The function $t \mapsto \mu_{t}(x)$ on $(0, \infty)$ into [0, $\left.\infty\right)$ is denoted by $\mu(x)$. See [13] (also $[32,42])$ for details on generalized $s$-numbers. Given $x \in \widetilde{\mathscr{M}}_{\text {sa }}$, we define the functions $\lambda(x)$ and $\check{\lambda}(x)$ on $(0, \tau(1))$ into $\mathbf{R}$ by

$$
\begin{aligned}
& \lambda_{t}(x)=\inf \left\{s \in \mathbf{R}: \tau\left(e_{(s, \infty)}(x)\right) \leq t\right\}, \\
& \check{\lambda}_{t}(x)=\sup \left\{s \in \mathbf{R}: \tau\left(e_{(-\infty, s)}(x)\right) \leq t\right\},
\end{aligned}
$$

and call them the spectral scales of $x$ following [33]. Obviously, if $x \in \widetilde{\mathscr{M}}_{+}$, then $\lambda_{t}(x)=\mu_{t}(x)$ for $0<t<\tau(1)$. The spectral scale $\lambda(x)$ (resp. $\check{\lambda}(x)$ ) is nonincreasing (resp. nondecreasing) and right-continuous. Note that $\grave{\lambda}(x)=$ $-\lambda(-x)$. Also, when $\tau(1)<\infty$, note that $\dot{\lambda}_{t}(x)=\lambda_{\tau(1)-t-0}(x)$ for $0<t<$ $\tau(1)$. As given in [18, Remark 6.1] (see also [20,33]), elementary properties of $\lambda(x)$ are analogous to those of $\mu(x)$.

When $\mathscr{M}$ is commutative, i.e. $\mathscr{M}=L^{\infty}(\Omega)$ and $\tau(f)=\int_{\Omega} f d m$ on a localizable measure space $(\Omega, m), \widetilde{\mathscr{M}}_{\text {sa }}$ consists of all real measurable functions $f$ on $\Omega$ bounded except on $m$-finite sets. For such $f, \lambda(f)$ is nothing but the decreasing rearrangement $f^{*}$ of $f$ (see e.g. [5]):

$$
\lambda_{t}(f)=f^{*}(t)=\inf \{s \in \mathbf{R}: m(\{\omega \in \Omega: f(\omega)>s\}) \leq t\}, \quad 0<t<m(\Omega) .
$$

When $\tau(1)=\infty$, the essential spectrum $\sigma_{e}(x)$ of $x \in \widetilde{\mathscr{M}}_{\mathrm{sa}}$ is given by

$$
\sigma_{e}(x)=\left\{s \in \mathbf{R}: \tau\left(e_{(s-\varepsilon, s+\varepsilon)}(x)\right)=\infty, \varepsilon>0\right\},
$$

which is a nonempty compact subset of $\mathbf{R}$.

An interval of $\mathbf{R}$ is considered as the measure space with Lebesgue measure. For $\alpha, \beta \in \mathbf{R}$, let $\alpha \vee \beta=\max \{\alpha, \beta\}, \alpha \wedge \beta=\min \{\alpha, \beta\}$ and $\alpha_{+}=\alpha \vee 0$ as usual. The characteristic function of a set $E$ is denoted by $\chi_{E}$. Let $x=x_{+}-x_{-}$ be the Jordan decomposition of $x \in \widetilde{\mathscr{M}}_{\mathrm{sa}}$. if

Given $x, y \in \widetilde{\mathscr{M}}_{+}$, we say that $x$ is submajorized by $y$, in notation $x \prec y$,

$$
\int_{0}^{s} \lambda_{t}(x) d t \leq \int_{0}^{s} \lambda_{t}(y) d t, \quad 0<s<\tau(1),
$$

and that $x$ is supermajorized by $y$, in notation $x \dot{<} y$, if

$$
\int_{s}^{\tau(1)} \lambda_{t}(x) d t \geq \int_{s}^{\tau(1)} \lambda_{t}(y) d t, \quad 0<s<\tau(1) .
$$

Furthermore when $x, y \in L^{1}(\mathscr{M})_{+}$, we say that $x$ is majorized by $y$, in notation $x \prec y$, if $x \prec y$ and $\tau(x)=\tau(y)$ (equivalently if $x \prec y$ and $x \prec y$ ). If $\tau(1)<\infty$, then these definitions of $x \prec y, x<y$, and $x<y$ are available to $x, y \in L^{1}(\mathscr{M})_{\text {sa }}$ too. The (sub)majorization in semifinite von Neumann algebras was discussed in [18-20].

The following properties of spectral scales are easily seen by definition or by the corresponding results for generalized $s$-numbers. 
(1) For $x \in \widetilde{\mathscr{M}}_{\mathrm{sa}}$ with $\tau(1)=\infty$, let $\Xi=\max \sigma_{e}(x)$ and $\xi=\min \sigma_{e}(x)$. Then $\lim _{t \rightarrow \infty} \lambda_{t}(x)=\Xi, \lim _{t \rightarrow \infty} \check{\lambda}_{t}(x)=\xi, \lambda(x)=\mu\left((x-\Xi)_{+}\right)+\Xi$ and $\check{\lambda}(x)=\xi-\mu\left((x-\xi)_{-}\right)$.

(2) If $\tau(1)=\infty$ and $x \in \widetilde{\mathscr{M}}_{\mathrm{sa}}$, then $x \in \widetilde{\mathfrak{S}}_{\mathrm{sa}}$ if and only if $\lim _{t \rightarrow \infty} \lambda_{t}(x)=$ $\lim _{t \rightarrow \infty} \check{\lambda}_{t}(x)=0$, i.e. $\sigma_{e}(x)=\{0\} \quad$ (cf. [13, Proposition 3.2]). Hence $\lambda(x)=$ $\mu\left(x_{+}\right)=\lambda\left(x_{+}\right)$and $\check{\lambda}(x)=-\mu\left(x_{-}\right)=-\lambda\left(x_{-}\right)$for $x \in \widetilde{\mathfrak{S}}_{\mathrm{sa}}$ with $\tau(1)=\infty$.

(3) If $\tau(1)<\infty$ and $x \in L^{1}(\mathscr{M})_{\mathrm{sa}}$, then $\tau(x)=\int_{0}^{\tau(1)} \lambda_{t}(x) d t$ (see [20, Proposition 1.1]). If $\tau(1)=\infty$ and $x \in L^{1}(\mathscr{M})_{\mathrm{sa}}$, then $\tau(x)=\int_{0}^{\infty} \lambda_{t}(x) d t+$ $\int_{0}^{\infty} \check{\lambda}_{t}(x) d t$ (cf. [13, Proposition 2.7]).

(4) If $x \in \widetilde{\mathscr{M}}_{\text {sa }}$, then $\int_{0}^{s} \lambda_{t}(x) d t<\infty$ for some (hence all) $0<s<\tau$ (1) if and only if $x_{+} \in\left(L^{1}(\mathscr{M})+\mathscr{M}\right)_{+}\left(=L^{1}(\mathscr{M})_{+}+\mathscr{M}_{+}\right)$(cf. [18, Proposition 1.2]).

In the next proposition, we further collect some properties of spectral scales which will be useful later. The analogous results for generalized $s$-numbers are found in [13].

Proposition 1.1. (1) If $x, y \in \mathscr{M}_{\mathrm{sa}}$, then for $0<t<\tau(1)$,

$$
\left|\lambda_{t}(x)-\lambda_{t}(y)\right| \leq\|x-y\|, \quad\left|\check{\lambda}_{t}(x)-\check{\lambda}_{t}(y)\right| \leq\|x-y\| .
$$

(2) If $\left\{x_{n}\right\}$ is a sequence in $\widetilde{\mathscr{M}}_{\mathrm{sa}}$ converging to $x$ in the measure topology, then for every $0<t<\tau(1)$,

$$
\lambda_{t}(x) \leq \liminf _{n \rightarrow \infty} \lambda_{t}\left(x_{n}\right), \quad \check{\lambda}_{t}(x) \geq \limsup _{n \rightarrow \infty} \check{\lambda}_{t}\left(x_{n}\right) .
$$

(3) If $x \in\left(L^{1}(\mathscr{M})+\mathscr{M}\right)_{\mathrm{sa}}$, then for every $0<s<\tau(1)$,

$$
\begin{aligned}
& \int_{0}^{s} \lambda_{t}(x) d t=\sup \{\tau(x a): a \in \mathscr{M}, 0 \leq a \leq 1, \tau(a)=s\}, \\
& \int_{0}^{s} \check{\lambda}_{t}(x) d t=\inf \{\tau(x a): a \in \mathscr{M}, 0 \leq a \leq 1, \tau(a)=s\} .
\end{aligned}
$$

If $\mathscr{M}$ is nonatomic, then the above equalities hold under the condition that a varies in the set of projections $e \in \mathscr{M}$ with $\tau(e)=s$.

Proof. Since $\check{\lambda}(x)=-\lambda(-x)$, it suffices to show the assertion for $\lambda(\cdot)$ in each of (1)-(3). If $x, y \in \widetilde{\mathscr{M}}_{\mathrm{sa}}$, then $\lambda_{t}(x) \leq \mu_{t}(x)$ and

$$
\lambda_{s+t}(x+y) \leq \lambda_{s}(x)+\lambda_{t}(y), \quad s, t, s+t \in(0, \tau(1)) .
$$

From these facts and [13, Lemma 3.1], (1) and (2) are easily shown (cf. [21, Lemma 3.2]). Let us prove (3). For every $\alpha \in \mathbf{R}$ and $a \in \mathscr{M}$ with $0 \leq a \leq 1$ and $\tau(a)=s$, by [13, Theorem 4.2] we get

$$
\begin{aligned}
\tau(x a) & \leq \tau\left((x-\alpha)_{+} a\right)+\alpha s \\
& \leq \int_{0}^{\infty} \mu_{t}\left((x-\alpha)_{+}\right) \mu_{t}(a) d t+\alpha s \\
& \leq \int_{0}^{s}\left\{\left(\lambda_{t}(x)-\alpha\right)_{+}+\alpha\right\} d t,
\end{aligned}
$$


because $\mu(a) \prec \chi_{(0, s)}$ as elements in $L^{1}(0, \infty)$ and $\mu_{t}\left((x-\alpha)_{+}\right)=\left(\lambda_{t}(x)-\alpha\right)_{+}$ for $0<t<\tau(1)$. If $\tau(1)=\infty$, then $\lambda(x)=(\lambda(x)-\alpha)_{+}+\alpha$ for $\alpha \leq$ $\max \sigma_{e}(x)$. On the other hand, if $\left.\tau, 1\right)<\infty$, then $x \in L^{1}(\mathscr{M})_{\text {sa }}$ and hence $\lambda(x) \in L^{1}(0, \tau(1))$, so that $(\lambda(x)-\alpha)_{+}+\alpha$ converges in $\|\cdot\|_{1}$ to $\lambda(x)$ as $\alpha \rightarrow-\infty$. Therefore $\tau(x a) \leq \int_{0}^{s} \lambda_{t}(x) d t$. Conversely let $r=\lambda_{s}(x)$. Given $\varepsilon>0$, since $\tau\left(e_{(r, \infty)}(x)\right) \leq s<\tau\left(e_{(r-\varepsilon, \infty)}(x)\right)$, we can choose a projection $q \in \mathscr{M}$ such that $q \leq e_{(r-\varepsilon, r]}(x)$ and $s^{\prime} \leq \tau(q)<\infty$ where $s^{\prime}=s-\tau\left(e_{(r, \infty)}(x)\right)$. Let

$$
a=e_{(r, \infty)}(x)+\frac{s^{\prime}}{\tau(q)} q .
$$

Then $0 \leq a \leq 1, \tau(a)=s$, and since

$$
a^{1 / 2} x a^{1 / 2} \geq x e_{(r, \infty)}(x)+\frac{(r-\varepsilon) s^{\prime}}{\tau(q)} q
$$

we get

$$
\begin{aligned}
\tau(x a) & \geq \tau\left((x-r)_{+}\right)+r \tau\left(e_{(r, \infty)}(x)\right)+(r-\varepsilon) s^{\prime} \\
& =\int_{0}^{\tau(1)}\left(\lambda_{t}(x)-r\right)_{+} d t+r \tau\left(e_{(r, \infty)}(x)\right)+(r-\varepsilon) s^{\prime} \\
& =\int_{0}^{s} \lambda_{t}(x) d t-\varepsilon s^{\prime} .
\end{aligned}
$$

Hence the desired equality is obtained. The last assertion is immediate from the above proof.

The following characterizations clarify the meaning of submajorization and supermajorization.

Proposition 1.2. Let $x, y \in \widetilde{\mathscr{M}}_{+}\left(\right.$resp. $x, y \in L^{1}(\mathscr{M})_{\text {sa }}$ when $\left.\tau(1)<\infty\right)$. Then:

(1) $x \prec y$ if and only if $\tau\left((x-\alpha)_{+}\right) \leq \tau\left((y-\alpha)_{+}\right)$for all $\alpha>0$ (resp. $\alpha \in \mathbf{R}$ ).

(2) $x \prec y$ if and only if $\tau(x \wedge \alpha) \geq \tau(y \wedge \alpha)$ for all $\alpha>0$ (resp. $\alpha \in \mathbf{R}$ ), where $x \wedge \alpha=x e_{(-\infty, \alpha]}(x)+\alpha e_{(\alpha, \infty)}(x)=x-(x-\alpha)_{+}$.

Proof. (1) See [20, Proposition 1.2].

(2) We show the case for $x, y \in \widetilde{\mathscr{M}}_{+}$(the proof for another case is analogous). First note that by [13, Lemma 2.5 and Proposition 2.7],

$$
\tau(x \wedge \alpha)=\int_{0}^{\tau(1)}\left(\lambda_{t}(x) \wedge \alpha\right) d t \leq \int_{s}^{\tau(1)} \lambda_{t}(x) d t+\alpha s
$$

for all $\alpha>0$ and $0<s<\tau(1)$. Suppose $x \prec y$. If $\alpha \geq \lambda_{0+}(x)$ or $0<\alpha \leq$ $\lim _{t \rightarrow \tau(1)} \lambda_{t}(x)$, then $\tau(x \wedge \alpha) \geq \tau(y \wedge \alpha)$ is immediate. When $\lim _{t \rightarrow \tau(1)} \lambda_{t}(x)<$ $\alpha<\lambda_{0+}(x)$, take an $s \in(0, \tau(1))$ such that $\lambda_{s}(x) \leq \alpha \leq \lambda_{s-0}(x)$. Then 
we have

$$
\begin{aligned}
\tau(x \wedge \alpha) & =\int_{s}^{\tau(1)} \lambda_{t}(x) d t+\alpha s \\
& \geq \int_{s}^{\tau(1)} \lambda_{t}(y) d t+\alpha s \geq \tau(y \wedge \alpha) .
\end{aligned}
$$

Conversely suppose that $\tau(x \wedge \alpha) \geq \tau(y \wedge \alpha)$ for all $\alpha>0$. For $0<s<\tau(1)$, let $\alpha=\lambda_{s}(y)$. Then we have

$$
\begin{aligned}
\int_{s}^{\tau(1)} \lambda_{t}(y) d t & =\tau(y \wedge \alpha)-\alpha s \\
& \leq \tau(x \wedge \alpha)-\alpha s \leq \int_{s}^{\tau(1)} \lambda_{t}(x) d t
\end{aligned}
$$

Proposition 1.3. If $\tau(1)<\infty$ and $x, y \in L^{1}(\mathscr{M})_{\mathrm{sa}}$, then $x \prec y$ if and only if $x_{+} \prec y_{+}, x_{-} \prec y_{-}$and $\tau(x)=\tau(y)$.

Proof. Suppose $x \prec y$, and hence $-x \prec-y$. Then $x_{+} \prec y_{+}$and $x_{-} \prec y_{-}$ follow from [20, Proposition 1.3]. Conversely suppose $x_{+} \prec y_{+}, x_{-} \prec y_{-}$and $\tau(x)=\tau(y)$. If either $y_{+}=0$ or $y_{-}=0$, then $x \prec y$ is readily verified. Assuming that $y_{+}$and $y_{-}$are nonzero, we choose an $r \in(0, \tau(1))$ such that $\lambda_{r}(y) \leq 0 \leq \lambda_{r-0}(y)$. When $0<s<r$,

$$
\int_{0}^{s} \lambda_{t}(x) d t \leq \int_{0}^{s} \lambda_{t}\left(x_{+}\right) d t \leq \int_{0}^{s} \lambda_{t}\left(y_{+}\right) d t=\int_{0}^{s} \lambda_{t}(y) d t,
$$

and when $r \leq s<\tau(1)$,

$$
\begin{aligned}
\int_{0}^{s} \lambda_{t}(x) d t & =\tau(x)-\int_{s}^{\tau(1)} \lambda_{t}(x) d t \\
& =\tau(x)+\int_{0}^{\tau(1)-s} \lambda_{t}(-x) d t \\
& \leq \tau(x)+\int_{0}^{\tau(1)-s} \lambda_{t}\left(x_{-}\right) d t \\
& \leq \tau(y)+\int_{0}^{\tau(1)-s} \lambda_{t}\left(y_{-}\right) d t \\
& =\tau(y)-\int_{s}^{\tau(1)} \lambda_{t}(y) d t=\int_{0}^{s} \lambda_{t}(y) d t
\end{aligned}
$$

so that $x \prec y$.

In view of this proposition, the notion of majorization $x \prec y$ is extended to each $x, y \in L^{1}(\mathscr{M})_{\text {sa }}$ whether $\tau(1)<\infty$ or $\tau(1)=\infty$ as follows: $x \prec y$ if $x_{+} \prec y_{+}, x_{-} \prec y_{-}$and $\tau(x)=\tau(y)$. This definition will be also justified from Theorems 2.3 and 3.5(4).

In the rest of this section, we establish some results on submajorization and supermajorization for real integrable functions on measure spaces. Those results will be used in $\S \S 2$ and 3 for functions on intervals (also vectors in $\mathbf{R}^{n}$ or $l^{1}$ ), 
while they are important by themselves in majorization theory for functions. Let $(\Omega, m)$ be a measure space and $L_{\mathbf{R}}^{1}(\Omega)$ (resp. $\left.L_{+}^{1}(\Omega)\right)$ be the set of all real (resp. positive) integrable functions on $\Omega$.

Proposition 1.4. (1) Let $f_{1}, f_{2}, g \in L_{+}^{1}(\Omega)$ (or $f_{1}, f_{2}, g \in L_{\mathbf{R}}^{1}(\Omega)$ when $m(\Omega)<\infty)$. If $f_{1} \leq f_{2}, f_{1} \prec g$ and $f_{2} \prec g$, then there exists an $h \in L_{\mathbf{R}}^{1}(\Omega)$ such that $f_{1} \leq h \leq f_{2}$ and $h \prec g$.

(2) Let $f, g \in L_{+}^{1}(\Omega)$ (or $f, g \in L_{\mathbf{R}}^{1}(\Omega)$ when $\left.m(\Omega)<\infty\right)$. Then $f \prec g$ if and only if there exists an $h \in L_{\mathbf{R}}^{1}(\Omega)$ such that $f \leq h$ and $h \prec g$.

(3) Let $f, g \in L_{+}^{1}(\Omega)$ (resp. $f, g \in L_{\mathbf{R}}^{1}(\Omega)$ when $m(\Omega)<\infty$ ). Then $f \dot{<} g$ if and only if there exists an $h \in L_{+}^{1}(\Omega)$ (resp. $h \in L_{\mathbf{R}}^{1}(\Omega)$ ) such that $h \leq f$ and $h \prec g$.

Note that (1) was given in [2, Theorem 1.7] for vectors in $\mathbf{R}^{n}$ and (2) was in [6, Theorem 1.1] when $m(\Omega)<\infty$. First let us prove the following lemma.

Lemma 1.5. Let $f_{1}, f_{2}, g$ be as in (1) of Proposition 1.4. If $\int_{\Omega} f_{1} d m \leq \gamma \leq$ $\int_{\Omega} g d m$, then there exists an $h \in L_{\mathbf{R}}^{1}(\Omega)$ such that $f_{1} \leq h \leq f_{2}, h \prec g$ and $\int_{\Omega} h d m=\gamma$.

Proof. We show the case for functions in $L_{+}^{1}(\Omega)$ (the proof for another case is analogous). If $\gamma=\int_{\Omega} f_{1} d m$, then $h=f_{1}$ is enough. Also if $\gamma=\int_{\Omega} f_{2} d m$, then $\int_{\Omega} f_{2} d m=\int_{\Omega} g d m$ and hence $f_{2} \prec g$, so $h=f_{2}$ is enough. Thus we may assume that $\int_{\Omega} f_{1} d m<\gamma<\int_{\Omega} f_{2} d m$. For $\alpha>0$, define $h_{\alpha} \in L_{\mathbf{R}}^{1}(\Omega)$ by

$$
h_{\alpha}=\left(f_{1}-\alpha\right)_{+}+\left(f_{2} \wedge \alpha\right) \text {; }
$$

then $f_{1} \leq h_{\alpha} \leq f_{2}$. Because $\int_{\Omega} h_{\alpha} d m$ is continuous in $\alpha>0$ and

$$
\lim _{\alpha \rightarrow 0} \int_{\Omega} h_{\alpha} d m=\int_{\Omega} f_{1} d m, \quad \lim _{\alpha \rightarrow \infty} \int_{\Omega} h_{\alpha} d m=\int_{\Omega} f_{2} d m,
$$

we can choose an $\alpha>0$ such that $\int_{\Omega} h_{\alpha} d m=\gamma$. When $\beta \geq \alpha$, since $f_{1} \prec g$ and $\left(h_{\alpha}-\beta\right)_{+}=\left(f_{1}-\beta\right)_{+}$, by Proposition 1.2(1) we get

$$
\int_{\Omega}\left(h_{\alpha}-\beta\right)_{+} d m \leq \int_{\Omega}(g-\beta)_{+} d m .
$$

When $0<\beta \leq \alpha$, since $f_{2} \dot{\prec} g$ and

$$
\left(h_{\alpha}-\beta\right)_{+}=h_{\alpha}-\left(h_{\alpha} \wedge \beta\right)=h_{\alpha}-\left(f_{2} \wedge \beta\right),
$$

by Proposition 1.2(2) we get

$$
\begin{aligned}
\int_{\Omega}\left(h_{r}-\beta\right)_{+} d m & =\gamma-\int_{\Omega}\left(f_{2} \wedge \beta\right) d m \\
& \leq \int_{\Omega}\{g-(g \wedge \beta)\} d m=\int_{\Omega}(g-\beta)_{+} d m .
\end{aligned}
$$

Therefore Proposition 1.2(1) implies $h_{c} \prec g$. 
Proof of Proposition 1.4. (1) follows from Lemma 1.5 by taking $\gamma=\int_{\Omega} g d m$. The "only if" parts of (2) and (3) follow by applying Lemma 1.5 to $f_{1}=f$, $f_{2}=f \vee g$ and to $f_{1}=f \wedge g, f_{2}=f$, respectively, together with $\gamma=\int_{\Omega} g d m$. The "if" parts are obvious.

Proposition 1.6. If $m(\Omega)=\infty$ and $f, g \in L_{\mathbf{R}}^{1}(\Omega)$, then the following conditions are equivalent:

(i) there exists an $h \in L_{\mathbf{R}}^{1}(\Omega)$ such that $f \leq h$ and $h \prec g$;

(ii) for every $s>0$,

$$
\begin{gathered}
\int_{0}^{s}\left(f_{+}\right)^{*}(t) d t \leq \int_{0}^{s}\left(g_{+}\right)^{*}(t) d t \quad\left(\text { i.e. } f_{+} \prec g_{+}\right) \\
\int_{\Omega} f_{+} d m-\int_{s}^{\infty}\left(f_{-}\right)^{*}(t) d t \leq \int_{\Omega} g_{+} d m-\int_{s}^{\infty}\left(g_{-}\right)^{*}(t) d t .
\end{gathered}
$$

Proof. (i) $\Rightarrow$ (ii). Since $f_{+} \leq h_{+} \prec g_{+}$and $f_{-} \geq h_{-} \prec g_{-}$by definition of $h \prec g$ in case of $m(\Omega)=\infty$, for every $s>0$ we get

$$
\int_{0}^{s}\left(f_{+}\right)^{*}(t) d t \leq \int_{0}^{s}\left(h_{+}\right)^{*}(t) d t \leq \int_{0}^{s}\left(g_{+}\right)^{*}(t) d t
$$

and

$$
\begin{aligned}
\int_{\Omega} f_{+} d m-\int_{s}^{\infty}\left(f_{-}\right)^{*}(t) d t & \leq \int_{\Omega} h_{+} d m-\int_{s}^{\infty}\left(h_{-}\right)^{*}(t) d t \\
& =\int_{\Omega} h d m+\int_{0}^{s}\left(h_{-}\right)^{*}(t) d t \\
& \leq \int_{\Omega} g d m+\int_{0}^{s}\left(g_{-}\right)^{*}(t) d t \\
& =\int_{\Omega} g_{+} d m-\int_{s}^{\infty}\left(g_{-}\right)^{*}(t) d t
\end{aligned}
$$

(ii) $\Rightarrow$ (i). If $\int_{\Omega} f d m=\int_{\Omega} g d m$, then $f_{-} \prec g_{-}$holds as well, so $h=f$ is enough. Thus assume that $\int_{\Omega} f d m<\int_{\Omega} g d m$. When $\int_{\Omega} f_{+} d m \leq \int_{\Omega} g d m$, applying Lemma 1.5 to $f_{+}, f_{+} \vee g_{+}, g_{+}$and $\gamma=\int_{\Omega} g d m$, we obtain an $h \in L_{+}^{1}(\Omega)$ such that $f_{+} \leq h \prec g_{+}$and $\int_{\Omega} h d m=\int_{\Omega} g d m$. These imply $f \leq h \prec g$. Next, when $\int_{\Omega} f_{+} d m>\int_{\Omega} g d m$, for $\alpha>0$ we define $h_{\alpha} \in L_{\mathbf{R}}^{1}(\Omega)$ by $h_{\alpha}=f \vee(-\alpha)$. Because $\int_{\Omega} h_{\alpha} d m$ is continuous in $\alpha>0$ and

$$
\lim _{\alpha \rightarrow 0} \int_{\Omega} h_{\alpha} d m=\int_{\Omega} f_{+} d m, \quad \lim _{\alpha \rightarrow \infty} \int_{\Omega} h_{\alpha} d m=\int_{\Omega} f d m,
$$

we can choose an $\alpha>0$ such that $\int_{\Omega} h_{\alpha} d m=\int_{\Omega} g d m$. Now let $h=h_{\alpha}$ and take an $r>0$ such that $\left(f_{-}\right)^{*}(r) \leq \alpha \leq\left(f_{-}\right)^{*}(r-0)$. Then $h_{+}=f_{+}$and

$$
\left(h_{-}\right)^{*}(t)= \begin{cases}\alpha, & 0<t<r \\ \left(f_{-}\right)^{*}(t), & t \geq r .\end{cases}
$$


To show $f \leq h \prec g$, it remains to check $h_{-} \prec g_{-}$. When $s \geq r$,

$$
\begin{aligned}
\int_{0}^{s}\left(h_{-}\right)^{*}(t) d t & =\int_{\Omega} h_{+} d m-\int_{s}^{\infty}\left(h_{-}\right)^{*}(t) d t-\int_{\Omega} h d m \\
& =\int_{\Omega} f_{+} d m-\int_{s}^{\infty}\left(f_{-}\right)^{*}(t) d t-\int_{\Omega} g d m \\
& \leq \int_{\Omega} g_{+} d m-\int_{s}^{\infty}\left(g_{-}\right)^{*}(t) d t-\int_{\Omega} g d m \\
& =\int_{0}^{s}\left(g_{-}\right)^{*}(t) d t,
\end{aligned}
$$

and when $0<s<r$,

$$
\begin{aligned}
\int_{0}^{s}\left(h_{-}\right)^{*}(t) d t & =\alpha s=\frac{s}{r} \int_{0}^{r}\left(h_{-}\right)^{*}(t) d t \\
& \leq \frac{s}{r} \int_{0}^{r}\left(g_{-}\right)^{*}(t) d t \leq \int_{0}^{s}\left(g_{-}\right)^{*}(t) d t,
\end{aligned}
$$

completing the proof.

Either condition of Proposition 1.6 may serve as a definition of submajorization $f \prec g$ for $f, g \in L_{\mathbf{R}}^{1}(\Omega)$ with $m(\Omega)=\infty$.

\section{THE FINITE FACTOR CASE}

Throughout this section, let $\mathscr{M}$ be a finite factor with a faithful normal trace $\tau$. To estimate $\operatorname{dist}(x, \operatorname{co} \mathscr{U}(y))$ for $x, y \in \mathscr{M}_{\text {sa }}$, we begin with a special problem of characterizing the condition $x \in \overline{\mathrm{co}} \mathscr{U}(y)$.

Theorem 2.1. Assume that $\mathscr{M}$ is a finite factor. If $x, y \in \mathscr{M}_{\mathrm{sa}}$, then the following conditions are equivalent:

(i) $x \in \overline{\operatorname{co}} \mathscr{U}(y)$;

(ii) $x \in \overline{\mathrm{co}}^{\mathrm{w}} \mathscr{U}(y)$;

(iii) $x \prec y$.

To prove the theorem, we need the next lemma.

Lemma 2.2. Assume that $\mathscr{M}$ is a factor of type $\mathrm{II}_{1}$. Let $e, p_{1}, p_{2} \in \mathscr{M}$ be projections with $e=p_{1}+p_{2}$, and $\alpha_{1}, \alpha_{2}, \beta_{1}, \beta_{2} \in \mathbf{R}$. Let $x=\alpha_{1} p_{1}+\alpha_{2} p_{2}$ and $y=\beta_{1} p_{1}+\beta_{2} p_{2}$. if $\beta_{1} \geq \alpha_{1} \geq \alpha_{2} \geq \beta_{2}$ and $\left(\beta_{1}-\alpha_{1}\right) \tau\left(p_{1}\right)=\left(\alpha_{2}-\beta_{2}\right) \tau\left(p_{2}\right)$, then $x \in \overline{\operatorname{co}} \mathscr{U}_{e}(y)$.

Proof. Because $\left(\beta_{1}-\alpha_{1}\right) \tau\left(p_{1}\right)=0$ implies $x=y$, suppose $\left(\beta_{1}-\alpha_{1}\right) \tau\left(p_{1}\right)>0$. Let $r_{k}=\left(\alpha_{k}-\beta_{2}\right) /\left(\beta_{1}-\beta_{2}\right)$ for $k=1,2$; then $\alpha_{k}=r_{k} \beta_{1}+\left(1-r_{k}\right) \beta_{2}$ and $\left(1-r_{1}\right) \tau\left(p_{1}\right)=r_{2} \tau\left(p_{2}\right)$. For each $0<\delta<\tau\left(p_{1}\right) \wedge \tau\left(p_{2}\right)$, let $n_{k}=\left[\tau\left(p_{k}\right) / \delta\right]$, $n=n_{1}+n_{2}, m=n_{1} \wedge n_{2}$, and

$$
\rho_{i j}= \begin{cases}r_{1} /\left(n_{1}+1\right), & 1 \leq i, j \leq n_{1}, \\ \left(1-r_{1}\right) /\left(n_{2}+1\right), & 1 \leq i \leq n_{1}<j \leq n, \\ r_{2} /\left(n_{1}+1\right), & 1 \leq j \leq n_{1}<i \leq n, \\ \left(1-r_{2}\right) /\left(n_{2}+1\right), & n_{1}<i, j \leq n .\end{cases}
$$


Then $\rho_{i j} \geq 0, \sum_{j=1}^{n} \rho_{i j} \leq 1$ and $\sum_{i=1}^{n} \rho_{i j} \leq 1$, because

$$
\begin{gathered}
\frac{n_{1} r_{1}}{n_{1}+1}+\frac{n_{2} r_{2}}{n_{1}+1} \leq r_{1}+\frac{\tau\left(p_{2}\right) r_{2}}{\tau\left(p_{1}\right)}=1, \\
\frac{n_{1}\left(1-r_{1}\right)}{n_{2}+1}+\frac{n_{2}\left(1-r_{2}\right)}{n_{2}+1} \leq \frac{\tau\left(p_{1}\right)\left(1-r_{1}\right)}{\tau\left(p_{2}\right)}+\left(1-r_{2}\right)=1 .
\end{gathered}
$$

By [2] or [18, Proposition 4.3], there is a doubly stochastic $n \times n$ matrix $\left[\tilde{\rho}_{i j}\right]$ such that $\left[\tilde{\rho}_{i j}\right] \geq\left[\rho_{i j}\right]$ entrywise, i.e. $\tilde{\rho}_{i j} \geq \rho_{i j}, \sum_{j=1}^{n} \tilde{\rho}_{i j}=1$ and $\sum_{i=1}^{n} \tilde{\rho}_{i j}=$ 1. Define $\tilde{\alpha}_{i}=\sum_{j=1}^{n} \tilde{\rho}_{i j} \tilde{\beta}_{j}, 1 \leq i \leq n$, where $\tilde{\beta}_{j}=\beta_{1}$ for $1 \leq j \leq n_{1}$ and $\tilde{\beta}_{j}=\beta_{2}$ for $n_{1}<j \leq n$. When $1 \leq i \leq n_{1}$ and $k=1$, or when $n_{1}<i \leq n$ and $k=2$, we get

$$
\begin{aligned}
\left|\tilde{\alpha}_{i}-\alpha_{k}\right|= & \left|\sum_{j=1}^{n_{1}}\left(\tilde{\rho}_{i j}-\frac{n_{1}+1}{n_{1}} \rho_{i j}\right) \beta_{1}+\sum_{j=n_{1}+1}^{n}\left(\tilde{\rho}_{i j}-\frac{n_{2}+1}{n_{2}} \rho_{i j}\right) \beta_{2}\right| \\
\leq & \left|\sum_{j=1}^{n_{1}}\left(\tilde{\rho}_{i j}-\rho_{i j}\right) \beta_{1}+\sum_{j=n_{1}+1}^{n}\left(\tilde{\rho}_{i j}-\rho_{i j}\right) \beta_{2}\right| \\
& +\left|\sum_{j=1}^{n_{1}} \frac{1}{n_{1}} \rho_{i j} \beta_{1}+\sum_{j=n_{1}+1}^{n} \frac{1}{n_{2}} \rho_{i j} \beta_{2}\right| \\
\leq & \left(1-\sum_{j=1}^{n} \rho_{i j}\right)\|y\|+\frac{1}{m} \sum_{j=1}^{n} \rho_{i j}\|y\| \\
\leq & \frac{2}{m}\|y\| .
\end{aligned}
$$

Because Birkhoff's theorem [3] asserts that $\left[\tilde{\rho}_{i j}\right]$ is a convex combination of permutation $n \times n$ matrices, there are permutations $\pi_{1}, \ldots, \pi_{K}$ of $\{1, \ldots, n\}$ and $\lambda_{1}, \ldots, \lambda_{K}>0$ with $\sum_{k=1}^{K} \lambda_{k}=1$ such that $\tilde{\alpha}_{i}=\sum_{k=1}^{K} \lambda_{k} \tilde{\beta}_{\pi_{k}(i)}$ for $1 \leq i \leq n$.

Now let $p_{1}=\sum_{i=1}^{n_{1}} q_{i}+e_{1}$ and $p_{2}=\sum_{i=n_{1}+1}^{n} q_{i}+e_{2}$ be partitions of $p_{1}$ and $p_{2}$ into projections in $\mathscr{M}$ with $\tau\left(q_{i}\right)=\delta, 1 \leq i \leq n$, and define

$$
y_{0}=\sum_{i=1}^{n} \tilde{\alpha}_{i} q_{i}+\beta_{1} e_{1}+\beta_{2} e_{2} .
$$

Choosing $u_{k} \in \mathscr{U}_{e}, 1 \leq k \leq K$, such that $u_{k} q_{\pi_{k}(i)} u_{k}^{*}=q_{i}$ for $1 \leq i \leq n$, $u_{k} e_{1} u_{k}^{*}=e_{1}$ and $u_{k} e_{2} u_{k}^{*}=e_{2}$, we have

$$
\begin{aligned}
y_{0} & =\sum_{k=1}^{K} \lambda_{k}\left(\sum_{i=1}^{n} \tilde{\beta}_{\pi_{k}(i)} q_{i}+\beta_{1} e_{1}+\beta_{2} e_{2}\right) \\
& =\sum_{k=1}^{K} \lambda_{k} u_{k} y u_{k}^{*} \in \operatorname{co} \mathscr{U}_{e}(y) .
\end{aligned}
$$


We can take partitions $p_{1}=\sum_{i=1}^{n_{1}} q_{i}^{(l)}+e_{1}^{(l)}$ and $p_{2}=\sum_{i=n_{1}+1}^{n} q_{i}^{(l)}+e_{2}^{(l)}$ as above for $1 \leq l \leq m$ so that $e_{1}^{(1)}, \ldots, e_{1}^{(l)}$ and $e_{2}^{(l)}, \ldots, e_{2}^{(l)}$ are mutually orthogonal. Then the above argument implies

$$
y_{l}=\sum_{i=1}^{n} \tilde{\alpha}_{i} q_{i}^{(l)}+\beta_{1} e_{1}^{(l)}+\beta_{2} e_{2}^{(l)} \in \operatorname{co} \mathscr{U}_{e}(y), \quad 1 \leq l \leq m
$$

and furthermore

$$
\begin{aligned}
\left\|x-\frac{1}{m} \sum_{l=1}^{m} y_{l}\right\|= & \| \frac{1}{m} \sum_{l=1}^{m}\left\{\sum_{i=1}^{n_{1}}\left(\alpha_{1}-\tilde{\alpha}_{i}\right) q_{i}^{(l)}+\sum_{i=n_{1}+1}^{n}\left(\alpha_{2}-\tilde{\alpha}_{i}\right) q_{i}^{(l)}\right. \\
& \left.\quad+\left(\alpha_{1}-\beta_{1}\right) e_{1}^{(l)}+\left(\alpha_{2}-\beta_{2}\right) e_{2}^{(l)}\right\} \| \\
\leq & \frac{1}{m} \sum_{l=1}^{m}\left\|\sum_{i=1}^{n_{1}}\left(\alpha_{1}-\tilde{\alpha}_{i}\right) q_{i}^{(l)}+\sum_{i=n_{1}+1}^{n}\left(\alpha_{2}-\tilde{\alpha}_{i}\right) q_{i}^{(l)}\right\| \\
& +\frac{1}{m}\left\|\left(\alpha_{1}-\beta_{1}\right) \sum_{l=1}^{m} e_{1}^{(l)}+\left(\alpha_{2}-\beta_{2}\right) \sum_{l=1}^{m} e_{2}^{(l)}\right\| \\
\leq & \frac{4}{m}\|y\| .
\end{aligned}
$$

Thus $x \in \overline{\mathrm{co}} \mathscr{U}_{e}(y)$ since $m \rightarrow \infty$ as $\delta \rightarrow 0$.

Proof of Theorem 2.1. (i) $\Rightarrow$ (ii) is obvious.

(ii) $\Rightarrow$ (iii). Let $\left\{x_{j}\right\}$ be a net in co $\mathscr{U}(y)$ such that $x_{j} \rightarrow x$ weakly. Let $0<s<\tau(1)$ and $a \in \mathscr{M}$ with $0 \leq a \leq 1$ and $\tau(a)=s$. Since

$$
\tau\left(x_{j} a\right) \leq \int_{0}^{s} \lambda_{t}\left(x_{j}\right) d t \leq \int_{0}^{s} \lambda_{t}(y) d t
$$

by Proposition 1.1(3), we get $\tau(x a) \leq \int_{0}^{s} \lambda_{t}(y) d t$, so that by Proposition 1.1(3) again, $\int_{0}^{s} \lambda_{t}(x) d t \leq \int_{0}^{s} \lambda_{t}(y) d t$. Also $\tau(x)=\lim _{j} \tau\left(x_{j}\right)=\tau(y)$, implying $x \prec$ $y$.

(iii) $\Rightarrow$ (i). Because this is well known when $\mathscr{M}=\mathbf{M}_{n}$ the $n \times n$ matrix algebra (see [2, Theorem 7.1] or [40, Theorem 12]), we assume that $\mathscr{M}$ is a factor of type $\mathrm{II}_{1}$ with $\tau(1)=1$. By the proof of [21, Theorem 4.3], there exists an increasing family $\left\{e_{t}: 0 \leq t \leq 1\right\}$ of projections in $\mathscr{M}$ such that $x=$ $\int_{0}^{1} \lambda_{t}(x) d e_{t}$ and $\tau\left(e_{t}\right)=t$ for $0 \leq t \leq 1$ (such a representation of $x$ is called the Schmidt decomposition [32]). Define $\tilde{y}=\int_{0}^{1} \lambda_{t}(y) d e_{t}$. Then $\lambda(\tilde{y})=\lambda(y)$ and hence $\tilde{y}$ is in the $\|\cdot\|$-closure of $\mathscr{U}(y)$ by [21, Lemma 4.1]. So we may let $y=\int_{0}^{1} \lambda_{t}(y) d e_{t}$ without loss of generality. Given $\varepsilon>0$, there exists a partition 
$0=t_{0}<t_{1}<\cdots<t_{n}=1$ such that

$$
\begin{aligned}
& \left\|x-\sum_{i=1}^{n} \alpha_{i}\left(e_{t_{i}}-e_{t_{i-1}}\right)\right\|<\varepsilon \quad \text { with } \alpha_{i}=\frac{1}{t_{i}-t_{i-1}} \int_{t_{i-1}}^{t_{i}} \lambda_{t}(x) d t, \\
& \left\|y-\sum_{i=1}^{n} \beta_{i}\left(e_{t_{i}}-e_{t_{i-1}}\right)\right\|<\varepsilon \quad \text { with } \beta_{i}=\frac{1}{t_{i}-t_{i-1}} \int_{t_{i-1}}^{t_{i}} \lambda_{t}(y) d t .
\end{aligned}
$$

Let $x_{0}=\sum_{i=1}^{n} \alpha_{i} p_{i}$ and $y_{0}=\sum_{i=1}^{n} \beta_{i} p_{i}$ where $p_{i}=e_{t_{i}}-e_{t_{i-1}}$. Then $x_{0} \prec y_{0}$, i.e. $\sum_{i=1}^{k} \alpha_{i} \tau\left(p_{i}\right) \leq \sum_{i=1}^{k} \beta_{i} \tau\left(p_{i}\right), 1 \leq k \leq n$, with equality for $k=n$. Now suppose that $\alpha_{i} \leq \beta_{i}$ for $1 \leq i<k$ and $\alpha_{k}>\beta_{k}$. Since

$$
\left(\alpha_{k}-\beta_{k}\right) \tau\left(p_{k}\right) \leq \sum_{i=1}^{k-1}\left(\beta_{i}-\alpha_{i}\right) \tau\left(p_{i}\right),
$$

we have

$$
\left(\alpha_{k}-\beta_{k}\right) \tau\left(p_{k}\right)=\left(\beta_{j}-\beta_{j}^{\prime}\right) \tau\left(p_{j}\right)+\sum_{i=j+1}^{k-1}\left(\beta_{i}-\alpha_{i}\right) \tau\left(p_{i}\right)
$$

for some $1 \leq j<k$ and $\alpha_{j} \leq \beta_{j}^{\prime} \leq \beta_{j}$. Define

$$
y_{0}^{\prime}=\sum_{i=1}^{j-1} \beta_{i} p_{i}+\beta_{j}^{\prime} p_{j}+\sum_{i=j+1}^{k} \alpha_{i} p_{i}+\sum_{i=k+1}^{n} \beta_{i} p_{i} .
$$

Then $x_{0} \prec y_{0}^{\prime}$, and the repeated use of Lemma 2.2 implies $y_{0}^{\prime} \in \overline{\operatorname{co}} \mathscr{U}\left(y_{0}\right)$. Writing $y_{0}^{\prime}=\sum_{i=1}^{n} \beta_{i}^{\prime} p_{i}$, we get $\alpha_{i} \leq \beta_{i}^{\prime}$ for $1 \leq i \leq k$. Thus, after repeating the above procedure finite times, we get a $y_{0}^{\prime \prime} \in \overline{\operatorname{co}} \mathscr{U}\left(y_{0}\right)$ such that $x_{0} \prec y_{0}^{\prime \prime}=$ $\sum_{i=1}^{n} \beta_{i}^{\prime \prime} p_{i}$ and $\alpha_{i} \leq \beta_{i}^{\prime \prime}$ for all $1 \leq i \leq n$, implying $x_{0}=y_{0}^{\prime \prime}$. Therefore $x_{0} \in \overline{\operatorname{co}} \mathscr{U}\left(y_{0}\right)$, so that

$$
\operatorname{dist}(x, \operatorname{co} \mathscr{U}(y)) \leq\left\|x-x_{0}\right\|+\left\|y-y_{0}\right\|<2 \varepsilon,
$$

which shows (i).

As for the unitary mixing in $L^{p}(\mathscr{M})_{\text {sa }}$, we have the next theorem.

Theorem 2.3. Assume that $\mathscr{M}$ is a finite factor. If $x, y \in L^{p}(\mathscr{M})_{\text {sa }}$ with $1 \leq$ $p<\infty$, then $x \in \overline{\mathrm{co}}^{L^{\prime \prime}} \mathscr{U}(y)$ if and only if $x \prec y$.

Proof. The case $p=1$ was given in [18, Theorem 2.5(1) and Remark 6.1] (also [23]). In case of $1<p<\infty$, since $\overline{\mathrm{co}}^{L^{\prime \prime}} \mathscr{U}(y)=\overline{\mathrm{co}}^{m} \mathscr{U}(y)$ by the proof of [18, Theorem 2.5(3)], it follows that $\overline{\mathrm{co}}^{L^{p}} \mathscr{U}(y)=\overline{\mathrm{co}}^{L^{1}} \mathscr{U}(y)$. This shows the theorem.

We now proceed to obtain the formulas of $\operatorname{dist}(x, \operatorname{co} \mathscr{U}(y))$ for $x, y \in \mathscr{M}_{\text {sa }}$ and of $\operatorname{dist}_{L^{1}}(x, \operatorname{co} \mathscr{U}(y))$ for $x, y \in L^{1}(\mathscr{M})_{\text {sa }}$ in terms of the spectral scales of $x$ and $y$. 
Theorem 2.4. Assume that $\mathscr{M}$ is a finite factor. If $x, y \in \mathscr{M}_{\mathrm{sa}}$, then

$$
\begin{array}{r}
\operatorname{dist}(x, \operatorname{co} \mathscr{U}(y))=\max \left\{\sup _{0<s<\tau(1)} \frac{1}{s} \int_{0}^{s}\left(\lambda_{t}(x)-\lambda_{t}(y)\right) d t,\right. \\
\left.\sup _{0<s<\tau(1)} \frac{1}{s} \int_{0}^{s}\left(\check{\lambda}_{t}(y)-\check{\lambda}_{t}(x)\right) d t\right\},
\end{array}
$$

and there exists a $z \in \overline{\mathrm{co}} \mathscr{U}(y)$ such that $\|x-z\|=\operatorname{dist}(x, \operatorname{co} \mathscr{U}(y))$.

Proof. Let $\rho$ be the right-hand side of the equality in ouestion. First note that $\rho \geq|\tau(x)-\tau(y)| / \tau(1) \geq 0$. For every $z \in \operatorname{co} \mathscr{U}(y)$ and $0<s<\tau(1)$, by Proposition 1.1(1) and Theorem 2.1 we get

$$
\begin{aligned}
\|x-z\| & \geq \frac{1}{s} \int_{0}^{s}\left(\lambda_{t}(x)-\lambda_{t}(z)\right) d t \geq \frac{1}{s} \int_{0}^{s}\left(\lambda_{t}(x)-\lambda_{t}(y)\right) d t, \\
\|x-z\| & \geq \frac{1}{s} \int_{0}^{s}\left(\check{\lambda}_{t}(z)-\check{\lambda}_{t}(x)\right) d t \geq \frac{1}{s} \int_{0}^{s}\left(\check{\lambda}_{t}(y)-\check{\lambda}_{t}(x)\right) d t .
\end{aligned}
$$

These imply $\operatorname{dist}(x, \operatorname{co} \mathscr{U}(y)) \geq \rho$. If $\rho=0$, then $x \prec y$ and hence $x \in$ $\overline{\mathrm{co}} \mathscr{U}(y)$ by Theorem 2.1. In the type $\mathrm{II}_{1}$ case with $\tau(1)=1$, we may let $x=\int_{0}^{1} \lambda_{t}(x) d e_{t}$ and $y=\int_{0}^{1} \lambda_{t}(y) d e_{t}$ as in the proof (iii) $\Rightarrow$ (i) of Theorem 2.1. Because

$$
\begin{array}{ll}
\int_{0}^{s}\left(\lambda_{t}(x)-\rho\right) d t \leq \int_{0}^{s} \lambda_{t}(y) d t, & 0<s<1, \\
\int_{s}^{1}\left(\lambda_{t}(x)+\rho\right) d t \geq \int_{s}^{1} \lambda_{t}(y) d t, & 0<s<1,
\end{array}
$$

applying Proposition 1.4(1) to $f_{1}=\lambda(x)-\rho, f_{2}=\lambda(x)+\rho$ and $g=\lambda(y)$ in $L_{\mathbf{R}}^{1}(0,1)$, we obtain an $h \in L_{\mathbf{R}}^{1}(0,1)$ such that $\lambda(x)-\rho \leq h \leq \lambda(x)+\rho$ and $h \prec \lambda(y)$. Define $z=\int_{0}^{1} h(t) d e_{t}$; then $\|x-z\| \leq \rho$. Since $\lambda(z)=h^{*}$ as easily verified, we get $z \prec y$ and hence $z \in \overline{c o} \mathscr{U}(y)$ by Theorem 2.1. The case $\mathscr{M}=\mathbf{M}_{n}$ is analogously seen from Proposition 1.4(1) applied to vectors in $\mathbf{R}^{n}$.

Theorem 2.5. Assume that $\mathscr{M}$ is a finite factor. If $x, y \in L^{1}(\mathscr{M})_{\mathrm{sa}}$, then

$$
\operatorname{dist}_{L^{1}}(x, \operatorname{co} \mathscr{U}(y))=2 \sup _{0<s<\tau(1)} \int_{0}^{s}\left(\lambda_{t}(x)-\lambda_{t}(y)\right) d t-\tau(x-y),
$$

and there exists a $z \in \overline{\mathrm{co}}^{L^{\prime}} \mathscr{U}(y)$ such that $\|x-z\|_{1}=\operatorname{dist}_{L^{\prime}}(x, \operatorname{co} \mathscr{U}(y))$.

Proof. Let $\rho$ be the right-hand side of the equality in question. For every $z \in \operatorname{co} \mathscr{U}(y)$ and $0<s<\tau(1)$, by [20, Lemma 2.2] and Theorem 2.3 we get

$$
\begin{aligned}
\|x-z\|_{1} & \geq\left\|\lambda_{(}(x)-\lambda(z)\right\|_{1} \\
& \geq 2 \int_{0}^{s}\left(\lambda_{t}(x)-\lambda_{t}(z)\right) d t-\tau(x-y) \\
& \geq 2 \int_{0}^{s}\left(\lambda_{t}(x)-\lambda_{t}(y)\right) d t-\tau(x-y) .
\end{aligned}
$$


Hence $\operatorname{dist}_{L^{1}}(x, \operatorname{co} \mathscr{U}(y)) \geq \rho$. Now assume that $\mathscr{M}$ is a factor of type $\mathrm{II}_{1}$ with $\tau(1)=1$, and let $x=\int_{0}^{1} \lambda_{t}(x) d e_{t}$ be the Schmidt decomposition. Also we may let $y=\int_{0}^{1} \lambda_{t}(y) d e_{t}$ (see [21, Lemma 4.1]). Choose an $r \in[0,1]$ such that

$$
\begin{aligned}
\rho & =2 \int_{0}^{r}\left(\lambda_{t}(x)-\lambda_{t}(y)\right) d t-\tau(x-y) \\
& =\int_{0}^{r}\left(\lambda_{t}(x)-\lambda_{t}(y)\right) d t+\int_{r}^{1}\left(\lambda_{t}(y)-\lambda_{t}(x)\right) d t .
\end{aligned}
$$

Since $\int_{0}^{s}\left(\lambda_{t}(x)-\lambda_{t}(y)\right) d t \leq \int_{0}^{r}\left(\lambda_{t}(x)-\lambda_{t}(y)\right) d t$ for all $0<s<1$, we have

$$
\begin{array}{ll}
\int_{s}^{r} \lambda_{t}(x) d t \geq \int_{s}^{r} \lambda_{t}(y) d t, & 0<s<r, \\
\int_{r}^{s} \lambda_{t}(x) d t \leq \int_{r}^{s} \lambda_{t}(y) d t, & r<s<1 .
\end{array}
$$

Let $f_{1}=\lambda(x)\left|(0, r), g_{1}=\lambda(y)\right|(0, r), f_{2}=\lambda(x) \mid(r, 1)$, and $g_{2}=\lambda(y) \mid(r, 1)$. Applying Proposition 1.4(3) to $f_{1}, g_{1} \in L_{\mathbf{R}}^{1}(0, r)$ and Proposition 1.4(2) to $f_{2}, g_{2} \in L_{\mathbf{R}}^{1}(r, 1)$, we obtain $h_{1} \in L_{\mathbf{R}}^{1}(0, r)$ and $h_{2} \in L_{\mathbf{R}}^{1}(r, 1)$ such that $f_{1} \geq h_{1} \prec g_{1}$ and $f_{2} \leq h_{2} \prec g_{2}$. Define

$$
z=\int_{0}^{r} h_{1}(t) d e_{t}+\int_{r}^{1} h_{2}(t) d e_{t} .
$$

Since

$$
\begin{aligned}
\left(h_{1}\right)^{*}(r-0) & \geq\left(g_{1}\right)^{*}(r-0)=g_{1}(r-0) \\
& \geq g_{2}(r+0)=\left(g_{2}\right)^{*}(0+) \geq\left(h_{2}\right)^{*}(0+),
\end{aligned}
$$

we get

$$
\lambda_{t}(z)= \begin{cases}\left(h_{1}\right)^{*}(t), & 0<t<r \\ \left(h_{2}\right)^{*}(t-r), & r<t<1 .\end{cases}
$$

This shows $z \prec y$, so that $z \in \overline{\mathrm{co}}^{L^{1}} \mathscr{U}(y)$ by Theorem 2.3. Moreover

$$
\begin{aligned}
\|x-z\|_{1} & =\int_{0}^{r}\left(f_{1}(t)-h_{1}(t)\right) d t+\int_{r}^{1}\left(h_{2}(t)-f_{2}(t)\right) d t \\
& =\int_{0}^{r}\left(f_{1}(t)-g_{1}(t)\right) d t+\int_{r}^{1}\left(g_{2}(t)-f_{2}(t)\right) d t=\rho .
\end{aligned}
$$

The proof in case of $\mathscr{M}=\mathbf{M}_{n}$ is analogous.

\section{THE INFINITE SEMIFINITE FACTOR CASE}

In this section, let $\mathscr{M}$ be an infinite semifinite factor with a faithful normal semifinite trace $\tau$. To characterize conditions such as $x \in \overline{\operatorname{co}} \mathscr{U}(y)$ for $x$, $y \in \mathscr{M}_{\text {sa }}$, we need the following lemmas. 
Lemma 3.1. Assume that $\mathscr{M}$ is an infinite semifinite factor.

(1) If $y \in\left(L^{1}(\mathscr{M})+\mathfrak{S}\right)_{+}$, then $0 \in \overline{\mathrm{co}}^{m} \mathscr{U}(y)$.

(2) If $y \in \mathfrak{S}_{+}$, then $0 \in \overline{\mathrm{co}} \mathscr{U}(y)$.

(3) If $y \in L^{1}(\mathscr{M})_{\text {sa }}$ and $\tau(y)=0$, then $0 \in \overline{\mathrm{co}}^{L^{1}} \mathscr{U}(y)$.

Proof. (1) For each integer $n \geq 1$, write $y=y_{n}^{\prime}+y_{n}^{\prime \prime}$ where $y_{n}^{\prime}=y e_{(1 / n, \infty)}(y)$ and $y_{n}^{\prime \prime}=y e_{[0,1 / n]}(y)$. Since $\tau\left(e_{(1 / n, \infty)}(y)\right)<\infty$, there are $u_{1}, \ldots, u_{n} \in \mathscr{U}$ such that $u_{i} e_{(1 / n, \infty)}(y) u_{i}^{*}, 1 \leq i \leq n$, are mutually orthogonal. Let $x_{n}=$ $n^{-1} \sum_{i=1}^{n} u_{i} y u_{i}^{*}$ and write $x_{n}=x_{n}^{\prime}+x_{n}^{\prime \prime}$ where $x_{n}^{\prime}=n^{-1} \sum_{i=1}^{n} u_{i} y_{n}^{\prime} u_{i}^{*}$ and $x_{n}^{\prime \prime}=n^{-1} \sum_{i=1}^{n} u_{i} y_{n}^{\prime \prime} u_{i}^{*}$. Then

$$
\mu_{t}\left(x_{n}\right) \leq \mu_{t}\left(x_{n}^{\prime}\right)+\left\|x_{n}^{\prime \prime}\right\| \leq \mu_{t}\left(x_{n}^{\prime}\right)+\left\|y_{n}^{\prime \prime}\right\| \leq \mu_{t}\left(x_{n}^{\prime}\right)+\frac{1}{n}
$$

for every $t>0$. Since

$$
e_{(s, \infty)}\left(x_{n}^{\prime}\right)=e_{(n s, \infty)}\left(\sum_{i=1}^{n} u_{i} y_{n}^{\prime} u_{i}^{*}\right)=\sum_{i=1}^{n} u_{i} e_{(n s, \infty)}\left(y_{n}^{\prime}\right) u_{i}^{*},
$$

we get $\tau\left(e_{(s, \infty)}\left(x_{n}^{\prime}\right)\right)=n \tau\left(e_{(n s, \infty)}\left(y_{n}^{\prime}\right)\right)$. Hence, for every $t>0$,

$$
\mu_{t}\left(x_{n}^{\prime}\right)=\frac{1}{n} \mu_{t / n}\left(y_{n}^{\prime}\right) \leq \frac{1}{n} \mu_{t / n}(y) \leq \frac{1}{t} \int_{0}^{t / n} \mu_{r}(y) d r \rightarrow 0
$$

as $n \rightarrow \infty$ by [18, Proposition 1.2], so that $\lim _{n \rightarrow \infty} \mu_{t}\left(x_{n}\right)=0$. Thus $x_{n} \rightarrow 0$ in the measure topology by [13, Lemma 3.1].

(2) is seen from the above proof of (1).

(3) In the type $\mathrm{II}_{\infty}$ case, we choose two increasing families $\left\{e_{t}: t \geq 0\right\}$ and $\left\{e_{t}^{\prime}: t \geq 0\right\}$ of projections in $\mathscr{M}$ such that $\tau\left(e_{t}\right)=\tau\left(e_{t}^{\prime}\right)=t$ for $t \geq 0, e \perp e^{\prime}$ and $\tau\left(\left(e+e^{\prime}\right)^{\perp}\right)=\infty$ where $e=\bigvee_{t \geq 0} e_{t}, e^{\prime}=\bigvee_{t \geq 0} e_{t}^{\prime}$, Define

$$
\tilde{y}=\int_{0}^{\infty} \lambda_{t}(y) d e_{t}+\int_{0}^{\infty} \check{\lambda}_{t}(y) d e_{t}^{\prime} .
$$

Since $\lambda(\tilde{y})=\lambda(y)$ and $\check{\lambda}(\tilde{y})=\check{\lambda}(y)$, it follows from [21, Lemma 4.1] that $\tilde{y}$ is in the $\|\cdot\|_{1}$-closure of $\mathscr{U}(y)$. So we may let $y=\tilde{y}$ without loss of generality. Let $s=\tau\left(y_{+}\right)$. Then $s=\tau\left(y_{-}\right)$since $\tau(y)=0$. Letting $s>0$, given $0<\varepsilon<s$ we take $r, r^{\prime} \in(0, \infty)$ such that

$$
\int_{r}^{\infty} \lambda_{t}(y) d t=-\int_{r^{\prime}}^{\infty} \check{\lambda}_{t}(y) d t=\varepsilon
$$

and write $y=y_{1}+y_{2}$ where

$$
\begin{aligned}
& y_{1}=\int_{0}^{r} \lambda_{t}(y) d e_{t}+\int_{0}^{r^{\prime}} \check{\lambda}_{t}(y) d e_{t}^{\prime}, \\
& y_{2}=\int_{r}^{\infty} \lambda_{t}(y) d e_{t}+\int_{r^{\prime}}^{\infty} \check{\lambda}_{t}(y) d e_{t}^{\prime} .
\end{aligned}
$$


Let $\alpha=\lambda_{r}(y) \wedge\left(-\check{\lambda}_{r^{\prime}}(y)\right)$; then $\alpha>0$. Now choose projections $p, p^{\prime} \in \mathscr{M}$ such that $p, p^{\prime} \leq\left(e+e^{\prime}\right)^{\perp}, p \perp p^{\prime}$ and $\tau(p)=\tau\left(p^{\prime}\right)=(s-\varepsilon) / \alpha$. Then $\alpha p \prec\left(y_{1}\right)_{+}$in $L^{1}\left(\mathscr{M}_{q}\right)_{+}$with $q=p+e$, and $\alpha p^{\prime} \prec\left(y_{1}\right)_{-}$in $L^{1}\left(\mathscr{M}_{q^{\prime}}\right)_{+}$with $q^{\prime}=p^{\prime}+e^{\prime}$. Hence $\alpha p \in \overline{\operatorname{co}}^{L^{\prime}} \mathscr{U}_{q}\left(\left(y_{1}\right)_{+}\right)$and $\alpha p^{\prime} \in \overline{\mathrm{co}}^{L^{\prime}} \mathscr{U}_{q^{\prime}}\left(\left(y_{1}\right)_{-}\right)$by [18, Theorem 2.5(1)], so that $\alpha p-\alpha p^{\prime} \in \overline{\mathrm{co}}^{L^{\prime}} \mathscr{U}\left(y_{1}\right)$. Exchanging $p$ and $p^{\prime}$, we get $\alpha p^{\prime}-\alpha p \in \overline{\mathrm{co}}^{L^{\prime}} \mathscr{U}\left(y_{1}\right)$ too. Therefore $0 \in \overline{\mathrm{co}}^{L^{1}} \mathscr{U}\left(y_{1}\right)$, which implies

$$
\operatorname{dist}_{L^{\prime}}(0, \operatorname{co} \mathscr{U}(y)) \leq\left\|y_{2}\right\|_{1}=2 \varepsilon,
$$

as desired. The proof in the type $I_{\infty}$ case is analogous.

Lemma 3.2. Assume that $\mathscr{M}$ is a $\sigma$-finite infinite semifinite factor. Let $y \in \mathscr{M}_{\mathrm{sa}}$ and $\xi, \Xi \in \mathbf{R}$ be such that $\xi \leq y \leq \Xi$. If $\tau\left(e_{\{\xi\}}(y)\right)=\tau\left(e_{\{\Xi\}}(y)\right)=\infty$, then $\overline{\mathrm{co}} \mathscr{U}(y)=\left\{x \in \mathscr{M}_{\mathrm{sa}}: \xi \leq x \leq \Xi\right\}$.

Proof. The assertion is obvious when $\xi=\Xi$, so let $\xi<\Xi$. Then, by taking $(y-\xi) /(\Xi-\xi)$ instead of $y$, we may let $\xi=0$ and $\Xi=1$. Now let $p \in \mathscr{M}$ be a projection. To prove $p \in \overline{\text { co }} \mathscr{U}(y)$, we can suppose $\tau\left(p^{\perp}\right)=\infty$ (consider $1-y$ and $p^{\perp}$ when $\left.\tau\left(p^{\perp}\right)<\infty\right)$. Since $\tau\left(e_{\{1\}}(y)\right)=\infty$, there is a projection $q$ such that $q \leq e_{\{1\}}(y), \tau(q)=\tau(p)$ and $\tau\left(e_{\{1\}}(y)-q\right)=\infty$. Since $p \sim q$, $p=u q u^{*}$ for some $u \in \mathscr{U}$. Moreover let $q_{0}=e_{(0,1]}(y)-q$, so $\tau\left(q_{0}\right)=\infty$. Since $\tau\left(e_{\{0\}}(y)\right)=\infty$, for each $n \geq 1$ we have a partition $e_{\{0\}}(y)=\sum_{i=1}^{n} q_{i}$ into projections with $\tau\left(q_{i}\right)=\infty$. For $1 \leq i \leq n$, take a $w_{i} \in \mathscr{M}$ such that $w_{i}^{*} w_{i}=q_{0}$ and $w_{i} w_{i}^{*}=q_{i}$, and let $u_{i}=w_{i}+w_{i}^{*}+\left(q_{0}+q_{i}\right)^{\perp}$. Then $u_{i} \in \mathscr{U}$ and

$$
u_{i} y u_{i}^{*}=u_{i}\left(q+y q_{0}\right) u_{i}^{*}=q+w_{i} y w_{i}^{*} .
$$

Defining $z=n^{-1} \sum_{i=1}^{n} u u_{i} y u_{i}^{*} u^{*}$ in co $\mathscr{U}(y)$, we get

$$
\|p-z\|=\left\|q-\frac{1}{n} \sum_{i=1}^{n} u_{i} y u_{i}^{*}\right\|=\left\|\frac{1}{n} \sum_{i=1}^{n} w_{i} y w_{i}^{*}\right\| \leq \frac{1}{n} .
$$

Thus the lemma follows from the fact that $\{x \in \mathscr{M}: 0 \leq x \leq 1\}$ is the $\|\cdot\|$ closed convex hull of all projections in $\mathscr{M}$.

Theorem 3.3. Assume that $\mathscr{M}$ is a $\sigma$-finite infinite semifinite factor. If $x, y \in$ $\mathscr{M}_{\text {sa }}$, then the following conditions are equivalent:

(i) $x \in \overline{\operatorname{co}} \mathscr{U}(y)$;

(ii) $x \in \overline{\mathrm{co}}^{\mathrm{w}} \mathscr{U}(y)$;

(iii) $\int_{0}^{s} \lambda_{t}(x) d t \leq \int_{0}^{s} \lambda_{t}(y) d t$ and $\int_{0}^{s} \check{\lambda}_{t}(x) d t \geq \int_{0}^{s} \check{\lambda}_{t}(y) d t$ for all $s>0$;

(iv) $(x-\Xi)_{+} \prec(y-\Xi)_{+}$and $(x-\xi)_{-} \prec(y-\xi)_{-}$where $\Xi=\max \sigma_{e}(y)$ and $\xi=\min \sigma_{e}(y)$.

Moreover (ii)-(iv) are equivalent without the $\sigma$-finiteness assumption of $\mathscr{M}$. 
Proof. (ii) $\Rightarrow$ (iii). For every $s>0, \int_{0}^{s} \lambda_{t}(x) d t \leq \int_{0}^{s} \lambda_{t}(y) d t$ is shown by Proposition $1.1(3)$ as in the proof (ii) $\Rightarrow$ (iii) of Theorem 2.1. Similarly

$$
\int_{0}^{s} \check{\lambda}_{t}(x) d t \geq \int_{0}^{s} \lambda_{t}(y) d t .
$$

(iii) $\Leftrightarrow$ (iv). The following equalities are immediate:

$$
\begin{aligned}
\lambda\left((x-\Xi)_{+}\right) & =(\lambda(x)-\Xi)_{+}, & & \lambda\left((y-\Xi)_{+}\right)=\lambda(y)-\Xi, \\
\lambda\left((x-\xi)_{-}\right) & =(\xi-\check{\lambda}(x))_{+}, & & \lambda\left((y-\xi)_{-}\right)=\xi-\check{\lambda}(y) .
\end{aligned}
$$

Hence (iv) $\Rightarrow$ (iii) is readily seen. Conversely suppose (iii). Let $r=$ $\sup \left\{t>0: \lambda_{t}(x)>\Xi\right\} \quad\left(r=0\right.$ if $\lambda_{t}(x) \leq \Xi$ for all $\left.t>0\right)$. For every $s>0$, we have

$$
\begin{aligned}
\int_{0}^{s} \lambda_{t}\left((x-\Xi)_{+}\right) d t & =\int_{0}^{r \wedge s}\left(\lambda_{t}(x)-\Xi\right) d t \\
& \leq \int_{0}^{r \wedge s}\left(\lambda_{t}(y)-\Xi\right) d t \leq \int_{0}^{s} \lambda_{t}\left((y-\Xi)_{+}\right) d t .
\end{aligned}
$$

Hence $(x-\Xi)_{+} \prec(y-\Xi)_{+}$and similarly $(x-\xi)_{-} \prec(y-\xi)_{-}$.

(iv) $\Rightarrow$ (i). We divide the proof into four steps.

Step 1 . Given $\varepsilon>0$, define

$$
x^{\prime}=\Xi e_{(\Xi, \Xi+\varepsilon]}(x)+\xi e_{[\xi-\varepsilon, \xi)}(x)+x e_{E}(x)
$$

where $E=\mathbf{R} \backslash((\Xi, \Xi+\varepsilon] \cup[\xi-\varepsilon, \xi))$. Then $\left\|x-x^{\prime}\right\| \leq \varepsilon,\left(x^{\prime}-\Xi\right)_{+} \leq$ $(x-\Xi)_{+}$and $\left(x^{\prime}-\xi\right)_{-} \leq(x-\xi)_{-}$. Furthermore, since $(y-\Xi)_{+} \in \mathfrak{S}$ and $(x-\Xi)_{+} \prec(y-\Xi)_{+}$, we get $(x-\Xi)_{+} \in \mathfrak{S}$ by [18, Proposition 2.1], so that

$$
\tau\left(e_{(\Xi, \infty)}\left(x^{\prime}\right)\right)=\tau\left(e_{(\Xi+\varepsilon, \infty)}(x)\right)=\tau\left(e_{(\varepsilon, \infty)}\left((x-\Xi)_{+}\right)\right)<\infty .
$$

Similarly $\tau\left(e_{(-\infty, \xi)}\left(x^{\prime}\right)\right)<\infty$. Thus we can assume that $\tau\left(e_{(\Xi, \infty)}(x)\right)<\infty$ and $\tau\left(e_{(-\infty, \xi)}(x)\right)<\infty$.

Step 2. Suppose $\xi<\Xi$. Given $0<\varepsilon<(\Xi-\xi) / 2$, define

$$
y^{\prime}=\Xi e_{(\Xi-\varepsilon, \Xi)}(y)+\xi e_{(\xi, \xi+\varepsilon)}(y)+y e_{F}(y)
$$

where $F=\mathbf{R} \backslash((\Xi-\varepsilon, \Xi) \cup(\xi, \xi+\varepsilon))$. Then $\left\|y-y^{\prime}\right\| \leq \varepsilon, \Xi=\max \sigma_{e}\left(y^{\prime}\right)$, $\xi=\min \sigma_{e}\left(y^{\prime}\right),\left(y^{\prime}-\Xi\right)_{+}=(y-\Xi)_{+},\left(y^{\prime}-\xi\right)_{-}=(y-\xi)_{-}$, and

$$
\begin{gathered}
\tau\left(e_{[\Xi, \infty)}\left(y^{\prime}\right)\right)=\tau\left(e_{(\Xi-\varepsilon, \infty)}(y)\right)=\infty, \\
\tau\left(e_{(-\infty, \xi]}\left(y^{\prime}\right)\right)=\tau\left(e_{(-\infty, \xi+\varepsilon)}(y)\right)=\infty .
\end{gathered}
$$

Next suppose $\xi=\Xi$ and $\tau\left(e_{\{\Xi\}}(y)\right)<\infty$. Then $\tau\left(e_{(-\infty, \Xi)}(y)\right)=\infty$ or $\tau\left(e_{(\Xi, \infty)}(y)\right)=\infty$. Suppose $\tau\left(e_{(\Xi, \infty)}(y)\right)=\infty$ and let $s=\tau\left(e_{(\Xi, \infty)}(x)\right) \quad(<\infty$ by Step 1). Moreover let $r=\lambda_{s}(y)$; then $r>\Xi$. We readily check that $(x-\Xi)_{+} \prec(y-\Xi)_{+} e_{(r, \infty)}(y)$. Given $0<\varepsilon<r-\Xi$, define

$$
y^{\prime}=\Xi e_{(\Xi, \Xi+\varepsilon)}(y)+y e_{\mathbf{R} \backslash(\Xi, \Xi+\varepsilon)}(y) .
$$


Then $\left\|y-y^{\prime}\right\| \leq \varepsilon, \sigma_{e}\left(y^{\prime}\right)=\{\Xi\},(y-\Xi)_{+} e_{(r, \infty)}(y) \leq\left(y^{\prime}-\Xi\right)_{+},\left(y^{\prime}-\Xi\right)_{-}=$ $(y-\Xi)_{-}$, and $\tau\left(e_{\{\Xi\}}\left(y^{\prime}\right)\right)=\infty$. The argument is analogous when $\xi=\Xi$ and $\tau\left(e_{(-\infty, \Xi)}(y)\right)=\infty$. Thus we can assume that $\tau\left(e_{[\Xi, \infty)}(y)\right)=\tau\left(e_{(-\infty, \xi]}(y)\right)=$ $\infty$.

Step 3. From now on, let us prove (iv) $\Rightarrow$ (i) under the additional assumptions observed in Steps 1 and 2. We can take projections $p, p^{\prime}$ such that $p \perp$ $p^{\prime}, \tau(p)=\tau\left(p^{\prime}\right)=\infty, e_{(\Xi, \infty)}(y) \leq p \leq e_{[\Xi, \infty)}(y)$ and $e_{(-\infty, \xi)}(y) \leq p^{\prime} \leq$ $e_{(-\infty, \xi]}(y)$. Define functions $f, f^{\prime}$ on $(0, \infty)$ and $b, b^{\prime} \in \mathfrak{S}_{+}$by

$$
\begin{aligned}
f & =\lambda\left((x-\Xi)_{+}\right), & & b=(y-\Xi)_{+} p, \\
f^{\prime} & =\lambda\left((x-\xi)_{-}\right), & & b^{\prime}=(y-\xi)_{-} p^{\prime} .
\end{aligned}
$$

Then $f \prec \lambda(b)$ and $f^{\prime} \prec \lambda\left(b^{\prime}\right)$. Regarding $b$ (resp. $\left.b^{\prime}\right)$ as an element in $\mathscr{M}_{p}$ (resp. $\mathscr{M}_{p^{\prime}}$ ), let us show the existence of $a \in \overline{\text { co }} \mathscr{U}_{p}(b)$ (resp. $a^{\prime} \in \overline{\text { co }} \mathscr{U}_{p^{\prime}}\left(b^{\prime}\right)$ ) such that $f \prec \lambda(a)$ (resp. $\left.f^{\prime} \prec \lambda\left(a^{\prime}\right)\right)$. Since $f(t)=0$ for all $t \geq \tau\left(e_{(\Xi, \infty)}(x)\right)$, there is an $s \in[0, \infty)$ such that $\int_{0}^{\infty} f(t) d t=\int_{0}^{s} \lambda_{t}(b) d t$. Letting $r=\lambda_{s}(b)$, we get $\tau\left(e_{(r, \infty)}(b)\right) \leq s \leq \tau\left(e_{[r, \infty)}(b)\right)$. First assume that $\mathscr{M}$ is a factor of type $\mathrm{II}_{\infty}$. Since $e_{(r, \infty)}(b) \leq e_{(\Xi, \infty)}(y) \leq p$, we can choose a projection $q$ such that $e_{(r, \infty)}(b) \leq q \leq e_{(r, \infty)}(b), q \leq p$ and $\tau(q)=s$. Define $a=b q$. Because $0 \in \overline{\mathrm{co}} \mathscr{U}_{p-q}(b(p-q))$ by Lemma 3.1(2), it follows that $a \in \overline{\mathrm{co}} \mathscr{U}_{p}(b)$. Moreover, since $\lambda(a)=\chi_{(0, s)} \lambda(b)$, we get $f \prec \lambda(a)$. Next assume that $\mathscr{M}$ is a factor of type $\mathrm{I}_{\infty}$ (i.e. $\mathscr{M}=\mathbf{B}(\mathscr{H})$ with the canonical trace). Take an integer $m$ with $m \leq s<m+1$. We can choose projections $q$, $\tilde{q}$ such that $e_{(r, \infty)}(b) \leq q \leq \tilde{q} \leq e_{[r, \infty)}(b), \tilde{q} \leq p, \tau(q)=m$ and $\tau(\tilde{q})=m+1$. Applying Lemma 3.1(2) to $b(p-q)$ and $b(p-\tilde{q})$, we see that $b q$ and $b \tilde{q}$ are in $\overline{c o} \mathscr{U}_{p}(b)$. Define $a \in \overline{\mathrm{co}} \mathscr{U}_{p}(b)$ by

$$
a=(m+1-s) b q+(s-m) b \tilde{q} .
$$

Since $a=b q+r(s-m)(\tilde{q}-q)$, we get

$$
\lambda_{t}(a)= \begin{cases}\lambda_{t}(b), & 0<t<m, \\ r(s-m), & m \leq t<m+1, \\ 0, & t \geq m+1 .\end{cases}
$$

Hence $f \prec \lambda(a)$ is verified as in the proof of [18, Lemma 2.6(1)]. The existence of $a^{\prime}$ is analogously shown. Now define

$$
\tilde{y}=(a+\Xi p)+\left(-a^{\prime}+\xi p^{\prime}\right)+y\left(p+p^{\prime}\right)^{\perp} .
$$

Since $\tilde{y} p=a+\Xi p, y p=b+\Xi p$ and $\tilde{y} p^{\prime}=-a^{\prime}+\xi p^{\prime}, y p^{\prime}=-b^{\prime}+\xi p^{\prime}$, we get $\tilde{y} p \in \overline{\mathrm{co}} \mathscr{U}_{p}(y p)$ and $\tilde{y} p^{\prime} \in \overline{\mathrm{co}} \mathscr{U}_{p^{\prime}}\left(y p^{\prime}\right)$. Therefore $\tilde{y} \in \overline{\mathrm{co}} \mathscr{U}(y)$. Furthermore we have

$$
\begin{aligned}
(x-\Xi)_{+} & \prec(\tilde{y}-\Xi)_{+}, \quad(x-\xi)_{-} \prec(\tilde{y}-\xi)_{-}, \\
& \tau\left(e_{\{\Xi\}}(\tilde{y})\right)=\tau\left(e_{\{\xi\}}(\tilde{y})\right)=\infty .
\end{aligned}
$$


Step 4. Finally it suffices to show $x \in \overline{\mathrm{co}} \mathscr{U}(\tilde{y})$ for $\tilde{y}$ obtained in Step 3. Let $e=e_{(\Xi, \infty)}(x), e^{\prime}=e_{(-\infty, \xi)}(x)$ and $e^{\prime \prime}=\left(e+e^{\prime}\right)^{\perp}$. We can choose projections $q, q^{\prime}$ such that

$$
\begin{array}{rlrl}
e_{(\Xi, \infty)}(\tilde{y}) & \leq q \leq e_{[\Xi, \infty)}(\tilde{y}), & \tau(q)=\tau(e), \\
e_{(-\infty, \xi)}(\tilde{y}) \leq q^{\prime} \leq e_{(-\infty, \xi]}(\tilde{y}), & \tau\left(q^{\prime}\right)=\tau\left(e^{\prime}\right) .
\end{array}
$$

Then $e=u q u^{*}, e^{\prime}=u q^{\prime} u^{*}$ and $e^{\prime \prime}=u\left(q+q^{\prime}\right)^{\perp} u^{*}$ for some $u \in \mathscr{U}$. Taking $u \tilde{y} u^{*}$ instead of $\tilde{y}$, we may suppose $q=e$ and $q^{\prime}=e^{\prime}$. Since $(x-\Xi)_{+} \prec$ $(\tilde{y}-\Xi)_{+}, x e \prec \tilde{y} e$ as elements in $\left(\mathscr{M}_{e}\right)_{\mathrm{sa}}$. Since $(x-\xi)_{-} \prec(\tilde{y}-\xi)_{-},-x e^{\prime} \prec$ $-\tilde{y} e^{\prime}$ (equivalently $\left.x e^{\prime} \prec \tilde{y} e^{\prime}\right)$ in $\left(\mathscr{M}_{e^{\prime}}\right)_{\text {sa }}$. Hence we get $x e \in \overline{\operatorname{co}} \mathscr{U}_{e}(\tilde{y} e)$ and $x e^{\prime} \in \overline{\operatorname{co}} \mathscr{U}_{e^{\prime}}\left(\tilde{y} e^{\prime}\right)$ by Theorem 2.1. On the other hand, since $\xi \leq x e^{\prime \prime} \leq \Xi$, $\xi \leq \tilde{y} e^{\prime \prime} \leq \Xi$ and $\tau\left(e_{\{\Xi\}}(\tilde{y})^{\prime \prime}\right)=\tau\left(e_{\{\xi\}}(\tilde{y}) e^{\prime \prime}\right)=\infty$, we get $x e^{\prime \prime} \in \overline{\operatorname{co}} \mathscr{U}_{e^{\prime \prime}}\left(\tilde{y} e^{\prime \prime}\right)$ by Lemma 3.2. These altogether imply the desired conclusion. Thus we complete the proof of the main part.

Let us prove the last statement of the theorem. Because the $\sigma$-finiteness assumption is unnecessary in the above proof of (ii) $\Rightarrow$ (iii) $\Leftrightarrow$ (iv), it suffices to show (iii) $\Rightarrow$ (ii) when $\mathscr{M}$ is not $\sigma$-finite. We can choose a $\sigma$-finite infinite projection $e_{0} \in \mathscr{M}$ such that $e_{(-\infty, \xi)}(y) \vee e_{(\Xi, \infty)}(y) \leq e_{0}$ and $\xi, \Xi \in \sigma_{e}\left(e_{0} y e_{0}\right)$ where $\sigma_{e}\left(e_{0} y e_{0}\right)$ is defined as that for an element in $\mathscr{M}_{e_{0}}$. Given positive elements $\varphi_{1}, \ldots, \varphi_{N}$ in $M_{*}$, let $e=e_{0} \vee \bigvee_{i=1}^{N} s\left(\varphi_{i}\right)$ where $s\left(\varphi_{i}\right)$ is the support projection of $\varphi_{i}$. Then the projection $e$ is $\sigma$-finite. When $\lambda($ exe $), \lambda(e y e)$, $\check{\lambda}($ exe $)$ and $\check{\lambda}($ eye $)$ are defined as those for elements in $\mathscr{M}_{e}$, it follows from Proposition 1.1(3) and condition (iii) that for every $s>0$,

$$
\begin{aligned}
& \int_{0}^{s} \lambda_{t}(\text { exe }) d t \leq \int_{0}^{s} \lambda_{t}(x) d t \leq \int_{0}^{s} \lambda_{t}(y) d t=\int_{0}^{s} \lambda_{t}(\text { eye }) d t, \\
& \int_{0}^{s} \check{\lambda}_{t}(\text { exe }) d t \geq \int_{0}^{s} \check{\lambda}_{t}(x) d t \geq \int_{0}^{s} \check{\lambda}_{t}(y) d t=\int_{0}^{s} \check{\lambda}_{t}(\text { eye }) d t,
\end{aligned}
$$

since $\lambda(y)=\lambda($ eye $)$ and $\check{\lambda}(y)=\check{\lambda}($ eye $)$ from the choice of $e_{0}$. Hence, by the main part of the theorem applied to exe, eye $\in \mathscr{M}_{e}$, given $\varepsilon>0$ there are $v_{1}, \ldots, v_{K} \in \mathscr{U}_{e}$ and $\lambda_{1}, \ldots, \lambda_{K}>0$ with $\sum_{k=1}^{K} \lambda_{k}=1$ such that

$$
\| \text { exe }-\sum_{k=1}^{K} \lambda_{k} v_{k}(\text { eye }) v_{k}^{*} \| \leq \varepsilon \text {. }
$$

Let $u_{k}=v_{k}+e^{\perp}$ for $1 \leq k \leq K$. Then $u_{k} \in \mathscr{U}$ and

$$
\begin{gathered}
\left|\varphi_{i}\left(x-\sum_{k=1}^{K} \lambda_{k} u_{k} y u_{k}^{*}\right)\right|=\left|\varphi_{i}\left(e\left(x-\sum_{k=1}^{K} \lambda_{k} u_{k} y u_{k}^{*}\right) e\right)\right| \\
=\mid \varphi_{i}\left(\text { exe }-\sum_{k=1}^{K} \lambda_{k} v_{k}(\text { eye }) v_{k}^{*}\right) \mid \leq \varepsilon\left\|\varphi_{i}\right\|
\end{gathered}
$$

for all $1 \leq i \leq N$. This shows that (ii) holds. 
As for the unitary mixing in the measure topology, we have the following modification of Theorem 3.3.

Theorem 3.4. Assume that $\mathscr{M}$ is a $\sigma$-finite infinite semifinite factor. If $x, y \in$ $\left(L^{1}(\mathscr{M})+\mathscr{M}\right)_{\mathrm{sa}}$, then the following condition $\left(\mathrm{i}^{\prime}\right)$ is equivalent to each of (iii) and (iv) in Theorem 3.3:

(i') $x \in \overline{\mathrm{co}}^{m} \mathscr{U}(y)$.

Proof. $\left(\mathrm{i}^{\prime}\right) \Rightarrow$ (iii) is readily seen from Proposition 1.1(2) and (3). The proof of (iii) $\Rightarrow$ (iv) is the same as that of Theorem 3.3. (iv) $\Rightarrow\left(i^{\prime}\right)$ can be proved as (iv) $\Rightarrow$ (i) of Theorem 3.3 with obvious slight modifications. Indeed Steps 1 and 2 stand with no change. Step 3 is carried out by using Lemma 3.1(1). In Step 4, we may use Theorem 2.3 for $x e, \tilde{y} e \in L^{1}\left(\mathscr{M}_{e}\right)_{\text {sa }}$ and $x e^{\prime}, \tilde{y} e^{\prime} \in$ $L^{1}\left(\mathscr{M}_{e^{\prime}}\right)_{\text {sa }}$ in place of Theorem 2.1 .

In the main part of Theorem 3.3 and in Theorem 3.4, the assumption of $\sigma$-finiteness of $\mathscr{M}$ cannot be removed. For instance, let $\mathscr{M}=\mathbf{B}(\mathscr{H})$ where $\mathscr{H}$ is not separable, and $q$ be a projection with countable infinite dimension. Then condition (iii) of Theorem 3.3 obviously holds for $x=q^{\perp}$ and $y=q$. Hence $q^{\perp} \in \overline{\mathrm{co}}^{\mathrm{w}} \mathscr{U}(q)$, but $q^{\perp}$ is not in $\overline{\mathrm{co}} \mathscr{U}(q)$ (cf. [34, Remark 3.6]). However we have the next theorem without the $\sigma$-finiteness assumption.

Theorem 3.5. Assume that $\mathscr{M}$ is an infinite semifinite factor. Let $x, y \in \tilde{\mathscr{M}}_{\mathrm{sa}}$.

(1) If $y \in \mathfrak{S}$, then $x \in \overline{\mathrm{co}} \mathscr{U}(y)$ if and only if $x_{+} \prec y_{+}$and $x_{-} \prec y_{-}$.

(2) If $y \in L^{1}(\mathscr{M})+\mathfrak{S}$, then $x \in \overline{\mathrm{co}}^{m} \mathscr{U}(y)$ if and only if $x_{+} \prec y_{+}$and $x_{-} \prec y_{-}$.

(3) If $y \in L^{p}(\mathscr{M})$ with $1<p<\infty$, then $x \in \overline{\operatorname{co}}^{L^{p}} \mathscr{U}(y)$ if and only if $x_{+} \prec y_{+}$ and $x_{-} \prec y_{-}$.

(4) If $y \in L^{1}(\mathscr{M})$, then $x \in \overline{\mathrm{co}}^{L^{1}} \mathscr{U}(y)$ if and only if $x \prec y$, i.e. $x_{+} \prec y_{+}$, $x_{-} \prec y_{-}$and $\tau(x)=\tau(y)$.

Proof. If $y$ is in the class mentioned in each of (1)-(4) and if $x_{+} \prec y_{+}$and $x_{-} \prec y_{-}$, then $x$ is in the same class (see [18, Proposition 2.1]). Here note for (2) that $L^{1}(\mathscr{M})+\mathfrak{S}=\left(L^{1}(\mathscr{M})+\mathscr{M}\right) \cap \tilde{S}$. When we are restricted to $y \in \mathfrak{S}_{\text {sa }}$ in Theorem 3.3 and to $y \in\left(L^{1}(\mathscr{M})+\mathfrak{S}\right)_{\text {sa }}$ in Theorem 3.4, there is no use of Lemma 3.2 because $\Xi=\xi=0$. Hence (1) and (2) hold. If $y \in L^{p}(\mathscr{M})$ with $1<p<\infty$, then $\overline{\mathrm{co}}^{L^{p}} \mathscr{U}(y)=\overline{\mathrm{co}}^{m} \mathscr{U}(y)$ by the proof of [18, Theorem 2.5(3)]. Hence (3) follows from (2).

Finally let us prove (4). Let $x, y \in L^{1}(\mathscr{M})_{\mathrm{sa}}$ be such that $x_{+} \prec y_{+}, x_{-} \prec y_{-}$ and $\tau(x)=\tau(y)$. Assume in the following that $\mathscr{M}$ is a type $\mathrm{II}_{\infty}$ factor. We choose $\left\{e_{t}: t \geq 0\right\}$ and $\left\{e_{t}^{\prime}: t \geq 0\right\}$ as in the proof of Lemma 3.1(3). Then, by [21, Lemma 4.1], we may let

$$
y=\int_{0}^{\infty} \lambda_{t}(y) d e_{t}+\int_{0}^{\infty} \check{\lambda}_{t}(y) d e_{t}^{\prime},
$$


and also $e_{(0, \infty)}(|x|) \leq\left(e+e^{\prime}\right)^{\perp}$ where $e=\bigvee_{t \geq 0} e_{t}, e^{\prime}=\bigvee_{t \geq 0} e_{t}^{\prime}$. Let $s=$ $\tau\left(y_{+}\right)-\tau\left(x_{+}\right)$; then $0 \leq s=\tau\left(y_{-}\right)-\tau\left(x_{-}\right)$by assumption. Taking $r, r^{\prime} \in[0, \infty]$ such that

$$
\int_{r}^{\infty} \lambda_{t}(y) d t=-\int_{r^{\prime}}^{\infty} \check{\lambda}_{t}(y) d t=s,
$$

we write $y=y_{1}+y_{2}$ as in the proof of Lemma 3.1(3). Then $x_{+} \prec\left(y_{1}\right)_{+}$in $L^{1}\left(\mathscr{M}_{q}\right)_{+}$with $q=e_{(0, \infty)}(x)+e$, and $x_{-} \prec\left(y_{1}\right)_{-}$in $L^{1}\left(\mathscr{M}_{q^{\prime}}\right)_{+}$with $q^{\prime}=$ $e_{(-\infty, 0)}(x)+e^{\prime}$. Hence $x_{+} \in \overline{\mathrm{co}}^{L^{\prime}} \mathscr{U}_{q}\left(\left(y_{1}\right)_{+}\right)$and $x_{-} \in \overline{\mathrm{co}}^{L^{1}} \mathscr{U}_{q^{\prime}}\left(\left(y_{1}\right)_{-}\right)$by [18, Theorem 2.5(1)]. Furthermore, since $y_{2} \in L^{1}\left(\mathscr{M}_{q^{\prime \prime}}\right)_{\text {sa }}$ with $q^{\prime \prime}=\left(q+q^{\prime}\right)^{\perp}$ and $\tau\left(y_{2}\right)=0$, we get $0 \in \overline{\mathrm{co}}^{L^{1}} \mathscr{U}_{q^{\prime \prime}}\left(y_{2}\right)$ by Lemma 3.1(3). These imply $x \in$ $\overline{\mathrm{co}}^{L^{1}} \mathscr{U}(y)$. The converse is immediate from (2).

In the sequel of this section, we establish the formulas of $\operatorname{dist}(x, \operatorname{co} \mathscr{U}(y))$ for $x, y \in \mathscr{M}_{\mathrm{sa}}$ and of $\operatorname{dist}_{L^{1}}(x, \operatorname{co} \mathscr{U}(y))$ for $x, y \in L^{1}(\mathscr{M})_{\mathrm{sa}}$. The proof of the next theorem is rather easy by virtue of Theorem 3.3.

Theorem 3.6. Assume that $\mathscr{M}$ is a $\sigma$-finite infinite semifinite factor. If $x, y \in$ $\mathscr{M}_{\text {sa }}$, then

$$
\begin{array}{r}
\operatorname{dist}(x, \operatorname{co} \mathscr{U}(y))=\max \left\{0, \sup _{s>0} \frac{1}{s} \int_{0}^{s}\left(\lambda_{t}(x)-\lambda_{t}(y)\right) d t,\right. \\
\left.\sup _{s>0} \frac{1}{s} \int_{0}^{s}\left(\check{\lambda}_{t}(y)-\check{\lambda}_{t}(x)\right) d t\right\},
\end{array}
$$

and there exists a $z \in \overline{\mathrm{co}} \mathscr{U}(y)$ such that $\|x-z\|=\operatorname{dist}(x, \operatorname{co} \mathscr{U}(y))$.

Proof. Let $\rho$ be the right-hand side of the above equality. For every $z \in$ co $\mathscr{U}(y)$ and $s>0$, by Proposition 1.1(1) and Theorem 3.3 we have

$$
\|x-z\| \geq \frac{1}{s} \int_{0}^{s}\left(\lambda_{t}(x)-\lambda_{t}(z)\right) d t \geq \frac{1}{s} \int_{0}^{s}\left(\lambda_{t}(x)-\lambda_{t}(y)\right) d t,
$$

and similarly

$$
\|x-z\| \geq \frac{1}{s} \int_{0}^{s}\left(\check{\lambda}_{t}(y)-\check{\lambda}_{t}(x)\right) d t .
$$

Hence $\operatorname{dist}(x, \operatorname{co} \mathscr{U}(y)) \geq \rho$. Conversely define

$$
\begin{aligned}
z= & (x-\rho) e_{(\Xi+\rho, \infty)}(x)+\Xi e_{(\Xi, \Xi+\rho]}(x) \\
& +(x+\rho) e_{(-\infty, \xi-\rho)}(x)+\xi e_{[\xi-\rho, \xi)}(x)+x e_{[\xi, \Xi]}(x),
\end{aligned}
$$

where $\Xi=\max \sigma_{e}(y)$ and $\xi=\min \sigma_{e}(y)$. Then $x-\rho \leq z \leq x+\rho$, so $\|x-z\| \leq \rho$. Moreover it is easy to check that $\lambda(z) \leq(\lambda(x)-\rho) \vee \Xi$ and $\check{\lambda}(z) \geq(\check{\lambda}(x)+\rho) \wedge \xi$. For every $s>0$, because

$$
\begin{aligned}
& \int_{0}^{s}\left(\lambda_{t}(x)-\rho\right) d t \leq \int_{0}^{s} \lambda_{t}(y) d t, \\
& \int_{0}^{s}\left(\check{\lambda}_{t}(x)+\rho\right) d t \geq \int_{0}^{s} \check{\lambda}_{t}(y) d t,
\end{aligned}
$$


we get $\int_{0}^{s} \lambda_{t}(z) d t \leq \int_{0}^{s} \lambda_{t}(y) d t$ and $\int_{0}^{s} \check{\lambda}_{t}(z) d t \geq \int_{0}^{s} \check{\lambda}_{t}(y) d t$. Therefore $z \in$ $\overline{\mathrm{co}} \mathscr{U}(y)$ by Theorem 3.3 .

Theorem 3.7. Assume that $\mathscr{M}$ is an infinite semifinite factor. If $x, y \in$ $L^{1}(\mathscr{M})_{\text {sa }}$, then

$$
\begin{array}{r}
\operatorname{dist}_{L^{1}}(x, \operatorname{co} \mathscr{U}(y))=\max \left\{2 \sup _{s>0} \int_{0}^{s}\left(\lambda_{t}(x)-\lambda_{t}(y)\right) d t-\tau(x-y),\right. \\
\left.2 \sup _{s>0} \int_{0}^{s}\left(\check{\lambda}_{t}(y)-\check{\lambda}_{t}(x)\right) d t+\tau(x-y)\right\},
\end{array}
$$

and there exists a $z \in \overline{\mathrm{co}}^{L^{1}} \mathscr{U}(y)$ such that $\|x-z\|_{1}=\operatorname{dist}_{L^{1}}(x, \operatorname{co} \mathscr{U}(y))$.

Proof. Let $\rho$ be the right-hand side of the above equality. For every $z \in$ co $\mathscr{U}(y)$ and $s>0$, by [21, Theorem 4.4] and Theorem 3.5(4) we have

$$
\begin{aligned}
\|x-z\|_{1} & \geq\|\lambda(x)-\lambda(z)\|_{1}+\|\check{\lambda}(x)-\check{\lambda}(z)\|_{1} \\
& \geq 2 \int_{0}^{s}\left(\lambda_{t}(x)-\lambda_{t}(z)\right) d t-\tau\left(x_{+}-z_{+}\right)+\tau\left(x_{-}-z_{-}\right) \\
& \geq 2 \int_{0}^{s}\left(\lambda_{t}(x)-\lambda_{t}(y)\right) d t-\tau(x-y),
\end{aligned}
$$

and similarly

$$
\|x-z\|_{1} \geq 2 \int_{0}^{s}\left(\check{\lambda}_{t}(y)-\check{\lambda}_{t}(x)\right) d t+\tau(x-y) .
$$

Hence $\operatorname{dist}_{L^{\prime}}(x, \operatorname{co} \mathscr{U}(y)) \geq \rho$. We continue the proof in the type $\mathrm{II}_{\infty}$ case as before (the case $\mathscr{M}=\mathbf{B}(\mathscr{H})$ is analogous from Propositions 1.4 and 1.6 applied to vectors in $\mathbf{R}^{n}$ or $l^{1}$ ). Considering $-x,-y$ instead of $x, y$ if necessary, we may suppose that

$$
\rho=2 \sup _{s>0} \int_{0}^{s}\left(\lambda_{t}(x)-\lambda_{t}(y)\right) d t-\tau(x-y) .
$$

So there is an $r \in[0, \infty]$ such that

$$
\begin{aligned}
\rho= & 2 \int_{0}^{r}\left(\lambda_{t}(x)-\lambda_{t}(y)\right) d t-\tau(x-y) \\
= & \int_{0}^{r}\left(\lambda_{t}(x)-\lambda_{t}(y)\right) d t+\int_{r}^{\infty}\left(\lambda_{t}(y)-\lambda_{t}(x)\right) d t \\
& +\int_{0}^{\infty}\left(\check{\lambda}_{t}(y)-\check{\lambda}_{t}(x)\right) d t .
\end{aligned}
$$

Then, for every $s>0$, we have

$$
\begin{gathered}
\int_{0}^{s}\left(\lambda_{t}(x)-\lambda_{t}(y)\right) d t \leq \int_{0}^{r}\left(\lambda_{t}(x)-\lambda_{t}(y)\right) d t, \\
\int_{0}^{s}\left(\check{\lambda}_{t}(y)-\check{\lambda}_{t}(x)\right) d t \leq \int_{0}^{r}\left(\lambda_{t}(x)-\lambda_{t}(y)\right) d t-\tau(x-y),
\end{gathered}
$$


so that

$$
\begin{gathered}
\int_{s}^{r} \lambda_{t}(x) d t \geq \int_{s}^{r} \lambda_{t}(y) d t, \quad 0<s<r, \\
\int_{r}^{s} \lambda_{t}(x) d t \leq \int_{r}^{s} \lambda_{t}(y) d t, \quad r<s<\infty, \\
\int_{r}^{\infty}\left(\lambda_{t}(x)-\lambda_{t}(y)\right) d t \leq \int_{s}^{\infty}\left(\check{\lambda}_{t}(y)-\check{\lambda}_{t}(x)\right) d t, \quad s>0 .
\end{gathered}
$$

Let $f_{1}=\lambda(x)\left|(0, r), g_{1}=\lambda(y)\right|(0, r), f_{2}=\lambda(x)\left|(r, \infty), g_{2}=\lambda(y)\right|(r, \infty)$, $f_{3}=-\check{\lambda}(x)$ and $g_{3}=-\check{\lambda}(y)$. Here we regard $(0, r)(\operatorname{resp} .(r, \infty))$ as a void interval when $r=0$ (resp. $r=\infty$ ). Applying Proposition 1.4(3) to $f_{1}$, $g_{1} \in L_{+}^{1}(0, r)$, we obtain an $h_{1} \in L_{+}^{1}(0, r)$ such that $f_{1} \geq h_{1} \prec g_{1}$. Replacing $h_{1}$ by $\left(h_{1}\right)^{*}$, we may let $h_{1}=\left(h_{1}\right)^{*}$. Next define $f, g \in L_{\mathbf{R}}^{1}((r, \infty) \cup(-\infty, 0))$ by

$$
\begin{aligned}
& f(t)= \begin{cases}f_{2}(t), & r<t<\infty, \\
-f_{3}(-t), & -\infty<t<0,\end{cases} \\
& g(t)= \begin{cases}g_{2}(t), & r<t<\infty, \\
-g_{3}(-t), & -\infty<t<0 .\end{cases}
\end{aligned}
$$

Then it follows that $f$ and $g$ satisfy condition (ii) of Proposition 1.6. Hence Proposition 1.6 implies that there exists an $h \in L_{\mathbf{R}}^{1}((r, \infty) \cup(-\infty, 0))$ satisfying $f \leq h \prec g$. Define $h_{2} \in L_{+}^{1}(r, \infty)$ and $h_{3} \in L_{+}^{1}(0, \infty)$ by $h_{2}(t)=\left(h_{+}\right)^{*}(t-r)$ and $h_{3}=\left(h_{-}\right)^{*}$. Then $f \leq h \prec g$ implies that $f_{2} \leq h_{2} \prec g_{2}, f_{3} \geq h_{3} \prec g_{3}$, and

$$
\int_{r}^{\infty} h_{2}(t) d t-\int_{0}^{\infty} h_{3}(t) d t=\int_{r}^{\infty} g_{2}(t) d t-\int_{0}^{\infty} g_{3}(t) d t
$$

The rest of the proof is divided into two cases.

Case 1. Suppose $\tau\left(e_{[0, \infty)}(x)\right)=\infty$. Let $e=e_{[0, \infty)}(x)$ and $s=\tau\left(e^{\perp}\right)$ $(\in[0, \infty])$. Taking the Schmidt decompositions of $x_{+} \in L^{1}\left(\mathscr{M}_{e}\right)_{+}$and $x_{-} \in$ $L^{1}\left(\mathscr{M}_{e^{\perp}}\right)_{+}$as in the proof of [21, Theorem 4.3], we can represent $x$ as follows:

$$
x=\int_{0}^{\infty} \lambda_{t}(x) d e_{t}+\int_{0}^{s} \check{\lambda}_{t}(x) d e_{t}^{\prime},
$$

where $\left\{e_{t}: t \geq 0\right\}$ and $\left\{e_{t}^{\prime}: 0 \leq t<s\right\}$ are increasing families of projections such that $e_{t} \leq e, \tau\left(e_{t}\right)=t$ for $t \geq 0$, and $e_{t}^{\prime} \leq e^{\perp}, \tau\left(e_{t}^{\prime}\right)=t$ for $0 \leq t<s$. Since $h_{3} \leq f_{3}, h_{3}(t)=f_{3}(t)=0$ for all $t \geq s$. Define

$$
z=\int_{0}^{r} h_{1}(t) d e_{t}+\int_{r}^{\infty} h_{2}(t) d e_{t}-\int_{0}^{s} h_{3}(t) d e_{t}^{\prime} .
$$

Since $h_{1}(r-0) \geq h_{2}(r+0)$, we get $\lambda(z)\left|(0, r)=h_{1}, \lambda(z)\right|(r, \infty)=h_{2}$, and also $-\check{\lambda}(z)=h_{3}$. Hence $z_{+} \prec y_{+}, z_{-} \prec y_{-}$, and

$$
\begin{aligned}
\tau(z) & =\int_{0}^{r} h_{1}(t) d t+\int_{r}^{\infty} h_{2}(t) d t-\int_{0}^{\infty} h_{3}(t) d t \\
& =\int_{0}^{r} g_{1}(t) d t+\int_{r}^{\infty} g_{2}(t) d t-\int_{0}^{\infty} g_{3}(t) d t=\tau(y),
\end{aligned}
$$


so that $z \in \overline{\mathrm{co}}^{L^{1}} \mathscr{U}(y)$ by Theorem 3.5(4). Furthermore

$$
\begin{aligned}
\|x-z\|_{1}= & \int_{0}^{r}\left(f_{1}(t)-h_{1}(t)\right) d t+\int_{r}^{\infty}\left(h_{2}(t)-f_{2}(t)\right) d t \\
& +\int_{0}^{\infty}\left(f_{3}(t)-h_{3}(t)\right) d t \\
= & \int_{0}^{r}\left(f_{1}(t)-g_{1}(t)\right) d t+\int_{r}^{\infty}\left(g_{2}(t)-f_{2}(t)\right) d t \\
& +\int_{0}^{\infty}\left(f_{3}(t)-g_{3}(t)\right) d t \\
= & \rho .
\end{aligned}
$$

Case 2. Suppose $\tau\left(e_{[0, \infty)}(x)\right)<\infty$. Let $s=\tau\left(e_{[0, \infty)}(x)\right)$. Taking the Schmidt decompositions of $x_{+}$and $x_{-}$, we write

$$
x=\int_{0}^{s} \lambda_{t}(x) d e_{t}+\int_{0}^{\infty} \check{\lambda}_{t}(x) d e_{t}^{\prime},
$$

where $\left\{e_{t}: 0 \leq t \leq s\right\}$ and $\left\{e_{t}^{\prime}: t \geq 0\right\}$ are as in Case 1. Since $\lambda_{t}(x)=0$ for $t \geq s$, we can suppose $r \leq s$. Define $h^{\prime} \in L_{\mathbf{R}}^{1}(0, \infty)$ by

$$
h^{\prime}(t)=h_{2}(s+t)-h_{3}(t), \quad t>0
$$

and

$$
z=\int_{0}^{r} h_{1}(t) d e_{t}+\int_{r}^{s} h_{2}(t) d e_{t}+\int_{0}^{\infty} h^{\prime}(t) d e_{t}^{\prime} .
$$

Letting $z^{\prime}=\int_{0}^{\infty} h^{\prime}(t) d e_{t}^{\prime}$, we easily verify that $\lambda\left(z^{\prime}\right)=\left(h_{+}^{\prime}\right)^{*}$ and $-\check{\lambda}\left(z^{\prime}\right)=$ $\left(h_{-}^{\prime}\right)^{*}$. Since $\left(h_{+}^{\prime}\right)^{*}(t) \leq h_{2}(s+t)$ and $\left(h_{-}^{\prime}\right)^{*}(t) \leq h_{3}(t)$ for all $t>0$, we get $\lambda(z)\left|(0, r)=h_{1}, \lambda(z)\right|(r, s)=h_{2}|(r, s), \lambda(z)|(s, \infty) \leq h_{2} \mid(s, \infty)$, and $-\check{\lambda}(z) \leq h_{3}$. Therefore $z_{+} \prec y_{+}, z_{-} \prec y_{-}$, and

$$
\begin{aligned}
\tau(z) & =\int_{0}^{r} h_{1}(t) d t+\int_{r}^{s} h_{2}(t) d t+\int_{0}^{\infty} h^{\prime}(t) d t \\
& =\int_{0}^{r} h_{1}(t) d t+\int_{r}^{\infty} h_{2}(t) d t-\int_{0}^{\infty} h_{3}(t)=\tau(y),
\end{aligned}
$$

so that $z \in \overline{\operatorname{co}}^{L^{\prime}} \mathscr{U}(y)$ by Theorem 3.5(4). Furthermore, because $f_{3}+h^{\prime} \geq$ $f_{3}-h_{3} \geq 0$, we have

$$
\begin{aligned}
\|x-z\|_{1}= & \int_{0}^{r}\left(f_{1}(t)-h_{1}(t)\right) d t+\int_{r}^{s}\left(h_{2}(t)-f_{2}(t)\right) d t \\
& +\int_{0}^{\infty}\left(f_{3}(t)+h^{\prime}(t)\right) d t \\
= & \int_{0}^{r}\left(f_{1}(t)-h_{1}(t)\right) d t+\int_{r}^{\infty}\left(h_{2}(t)-f_{2}(t)\right) d t \\
& +\int_{0}^{\infty}\left(f_{3}(t)-h_{3}(t)\right) d t \\
= & \rho . \square
\end{aligned}
$$


When $\mathscr{M}$ is a semifinite factor, it is seen from Theorems 2.3, 3.5(3) and [21, Theorems 4.3, 4.4] that $\operatorname{dist}_{L^{p}}(x, \operatorname{co} \mathscr{U}(y))$ for $x, y \in L^{p}(\mathscr{M})_{\text {sa }}, 1<p<\infty$, is determined by the spectral scales of $x$ and $y$. However it seems that there is no simple formula for the $L^{p}$-distance.

\section{THE TYPE III FACTOR CASE}

The main task in this section is to estimate $\operatorname{dist}(x, \operatorname{co} \mathscr{U}(y))$ for normal operators $x, y \in \mathscr{M}$ and $\operatorname{dist}(\varphi, \operatorname{co} \mathscr{U}(\psi))$ for selfadjoint elements $\varphi, \psi \in$ $\mathscr{M}_{*}$ when $\mathscr{M}$ is a type III factor. To begin with, we characterize $x \in \overline{\operatorname{co}} \mathscr{U}(y)$.

Theorem 4.1. Assume that $\mathscr{M}$ is a $\sigma$-finite factor of type III. If $x, y \in \mathscr{M}$ are normal, then the following conditions are equivalent:

(i) $x \in \overline{\mathrm{co}} \mathscr{U}(y)$;

(ii) $x \in \overline{\mathrm{Co}}^{\mathrm{w}} \mathscr{U}(y)$;

(iii) $\sigma(x) \subseteq \operatorname{co} \sigma(y)$ where $\sigma(x), \sigma(y)$ are the spectra of $x, y$.

Moreover (ii) and (iii) are equivalent without the $\sigma$-finiteness assumption of $\mathscr{M}$. Proof. (ii) $\Rightarrow$ (iii). As is well known [16, Problem 171], we have $\overline{W(y)}=\operatorname{co} \sigma(y)$ by normality of $y$, where $\overline{W(y)}$ is the closure of the numerical range of $y$. Let $\left\{x_{j}\right\}$ be a net in co $\mathscr{U}(y)$ such that $x_{j} \rightarrow x$ weakly. Then

$$
(x \eta \mid \eta)=\lim _{j}\left(x_{j} \eta \mid \eta\right) \in \cos (y)
$$

for every $\eta \in \mathscr{H}$ with $\|\eta\|=1$, where $\mathscr{M}$ acts on $\mathscr{H}$ with the inner product $(\cdot \mid \cdot)$. Hence $\sigma(x) \subseteq \overline{W(x)} \subseteq \operatorname{co} \sigma(y)$.

(iii) $\Rightarrow$ (i). Suppose $\sigma(x) \subseteq \operatorname{co} \sigma(y)$. To prove $x \in \overline{c o} \mathscr{U}(y)$, it suffices to show the case when $x$ and $y$ have finite spectra. Indeed, given $\varepsilon>0$, there exist normal operators $x^{\prime}, y^{\prime} \in \mathscr{M}$ with finite spectra such that $\left\|x-x^{\prime}\right\|<\varepsilon$, $\left\|y-y^{\prime}\right\|<\varepsilon$ and $\sigma\left(x^{\prime}\right) \subseteq \operatorname{co} \sigma\left(y^{\prime}\right)$. So we write $x=\sum_{i=1}^{m} \alpha_{i} p_{i}$ and $y=$ $\sum_{j=1}^{n} \beta_{j} q_{j}$ where $p_{i}, q_{j}$ are nonzero projections with $\sum_{i=1}^{m} p_{i}=\sum_{j=1}^{n} q_{j}=1$, and $\alpha_{i}, \beta_{j} \in \mathbf{C}$. For $1 \leq j \leq n$, let $q_{j}=\sum_{i=1}^{m} q_{i j}$ be a partition into nonzero projections. By $\sigma$-finiteness of $\mathscr{M}$, we may suppose $p_{i}=\sum_{j=1}^{n} q_{i j}$ because there is a $w \in \mathscr{U}$ such that $p_{i}=w\left(\sum_{j=1}^{n} q_{i j}\right) w^{*}, 1 \leq i \leq m$. Then (iii) follows from

$$
\alpha_{i} p_{i} \in \overline{\operatorname{co}} \mathscr{U}_{p_{\imath}}\left(\sum_{j=1}^{n} \beta_{j} q_{i j}\right), \quad 1 \leq i \leq m .
$$

Hence it suffices to show that $\alpha 1 \in \overline{\mathrm{co}} \mathscr{U}(y)$ whenever $\alpha \in \operatorname{co}\left\{\beta_{1}, \ldots, \beta_{n}\right\}$. Let $\alpha=\sum_{j=1}^{n} \rho_{j} \beta_{j}$ with $\rho_{j} \geq 0, \sum_{j=1}^{n} \rho_{j}=1$. Choosing a doubly stochastic $n \times n$ matrix $\left[\rho_{i j}\right]$ with $\rho_{1 j}=\rho_{j}$, we define $\gamma_{i}=\sum_{j=1}^{n} \rho_{i j} \beta_{j}$ for $1 \leq i \leq n$ (hence $\gamma_{1}=\alpha$ ) and $y_{0}=\sum_{j=1}^{n} \gamma_{j} q_{j}$. By Birkhoff's theorem [3], there are permutations $\pi_{1}, \ldots, \pi_{K}$ of $\{1, \ldots, n\}$ and $\lambda_{1}, \ldots, \lambda_{K}>0$ with $\sum_{k=1}^{K} \lambda_{k}=$ 1 such that $\gamma_{j}=\sum_{k=1}^{K} \lambda_{k} \beta_{\pi_{k}(j)}$ for $1 \leq j \leq n$. Let $u_{1}, \ldots, u_{K} \in \mathscr{U}$ be such 
that $u_{k} q_{\pi_{k}(j)} u_{k}^{*}=q_{j}, 1 \leq j \leq n, 1 \leq k \leq K$. Then

$$
y_{0}=\sum_{k=1}^{K} \lambda_{k}\left(\sum_{j=1}^{n} \beta_{\pi_{k}(j)} q_{j}\right)=\sum_{k=1}^{K} \lambda_{k} u_{k} y u_{k}^{*} \in \operatorname{co} \mathscr{U}(y) .
$$

Now let $q_{1}=\sum_{i=2}^{n}\left(p_{i}^{\prime}+p_{i}^{\prime \prime}\right)$ and $q_{j}=q_{j}^{\prime}+q_{j}^{\prime \prime}, 2 \leq j \leq n$, be partitions into nonzero projections. Choose $u, v \in \mathscr{U}$ such that for $2 \leq j \leq n$,

$$
\begin{array}{ll}
u p_{j}^{\prime \prime} u^{*}=p_{j}^{\prime}+p_{j}^{\prime \prime}, & u p_{j}^{\prime} u^{*}=q_{j}^{\prime}, \quad u q_{j} u^{*}=q_{j}^{\prime \prime}, \\
v p_{j}^{\prime \prime} v^{*}=p_{j}^{\prime}+p_{j}^{\prime \prime}, & v p_{j}^{\prime} v^{*}=q_{j}^{\prime \prime}, \quad v q_{j} v^{*}=q_{j}^{\prime},
\end{array}
$$

and define a sequence $\left\{y_{l}\right\}$ in co $\mathscr{U}(y)$ by

$$
y_{l}=\frac{1}{2}\left(u y_{l-1} u^{*}+v y_{l-1} v^{*}\right), \quad l \geq 1 \text {. }
$$

Then an easy computation gives

$$
y_{l}=\alpha q_{1}+\sum_{j=2}^{n}\left\{\left(1-\frac{1}{2^{l}}\right) \alpha+\frac{\gamma_{j}}{2^{l}}\right\} q_{j},
$$

so that $\left\|y_{l}-\alpha 1\right\| \rightarrow 0$ as $l \rightarrow \infty$, as desired. Thus the proof of the main part is completed.

Let us prove the last statement of the theorem. We need to show (iii) $\Rightarrow$ (ii) when $\mathscr{M}$ is not $\sigma$-finite. To do so, it suffices to show the case when $x=$ $\sum_{i=1}^{m} \alpha_{i} p_{i}$ and $y=\sum_{j=1}^{n} \beta_{j} q_{j}$ as above. Repeating some $\alpha_{i}$ or $\beta_{j}$ if necessary, we may suppose $m=n$. Given positive elements $\varphi_{1}, \ldots, \varphi_{N}$ in $\mathscr{M}_{*}$ and $\varepsilon>0$, there are $\sigma$-finite projections $e_{1}, \ldots, e_{n} \in \mathscr{M}$ such that $e_{j} \leq p_{j}$ and $\varphi_{i}\left(e^{\perp}\right) \leq \varepsilon, 1 \leq i \leq N$, where $e=\sum_{j=1}^{n} e_{j}$. Also choose nonzero $\sigma$-finite projections $e_{1}^{\prime}, \ldots, e_{n}^{\prime} \in \mathscr{M}$ with $e_{j}^{\prime} \leq q_{j}$, and let $e^{\prime}=\sum_{j=1}^{n} e_{j}^{\prime}$. Then there exists a $w \in \mathscr{U}$ such that $e_{j}=w e_{j}^{\prime} w^{*}, 1 \leq j \leq n$, and $e^{\perp}=w e^{\perp} w^{*}$. Applying the main part to $x_{0}=\sum_{j=1}^{n} \alpha_{j} e_{j}$ and $y_{0}=\sum_{j=1}^{n} \beta_{j} e_{j}$ in $\mathscr{M}_{e}$, we can choose $v_{1}, \ldots, v_{K} \in \mathscr{U}_{e}$ and $\lambda_{1}, \ldots, \lambda_{K}>0$ with $\sum_{k=1}^{K} \lambda_{k}=1$ such that

$$
\left\|x_{0}-\sum_{k=1}^{K} \lambda_{k} v_{k} y_{0} v_{k}^{*}\right\|<\varepsilon \text {. }
$$

For $1 \leq k \leq K$, let $u_{k}=v_{k}+e^{\perp}$; then $u_{k} \in \mathscr{U}$ and

$$
\begin{aligned}
e\left(x-\sum_{k=1}^{K} \lambda_{k} u_{k} w y w^{*} u_{k}^{*}\right) e & =x_{0}-\sum_{k=1}^{K} \lambda_{k} v_{k} w e^{\prime} y e^{\prime} w^{*} v_{k}^{*} \\
& =x_{0}-\sum_{k=1}^{K} \lambda_{k} v_{k} y_{0} v_{k}^{*} .
\end{aligned}
$$

For each $1 \leq i \leq N$, because

$$
\begin{aligned}
\left|\varphi_{i}(z)\right| & \leq\left|\varphi_{i}(e z e)\right|+\left|\varphi_{i}\left(e^{\perp} z e\right)\right|+\left|\varphi_{i}\left(z e^{\perp}\right)\right| \\
& \leq\left\|\varphi_{i}\right\|\|e z e\|+\varphi_{i}\left(e^{\perp}\right)^{1 / 2} \varphi_{i}\left((z e)^{*} z e\right)^{1 / 2}+\varphi_{i}\left(z z^{*}\right)^{1 / 2} \varphi_{i}\left(e^{\perp}\right)^{1 / 2} \\
& \leq\left\|\varphi_{i}\right\|\|e z e\|+2 \varepsilon^{1 / 2}\left\|\varphi_{i}\right\|^{1 / 2}\|z\|, \quad z \in \mathscr{M},
\end{aligned}
$$


we get

$$
\left|\varphi_{i}\left(x-\sum_{k=1}^{K} \lambda_{k} u_{k} w y w^{*} u_{k}^{*}\right)\right| \leq \varepsilon\left\|\varphi_{i}\right\|+2 \varepsilon^{1 / 2}\left\|\varphi_{i}\right\|^{1 / 2}(\|x\|+\|y\|) .
$$

This shows that (ii) holds.

Theorem 4.2. Assume that $\mathscr{M}$ is a $\sigma$-finite factor of type III. If $x, y \in \mathscr{M}$ are normal, then

$$
\operatorname{dist}(x, \operatorname{co} \mathscr{U}(y))=\sup _{\alpha \in \sigma(x)} \operatorname{dist}(\alpha, \operatorname{co} \sigma(y)),
$$

and there exists a normal operator $z \in \overline{\mathrm{co}} \mathscr{U}(y)$ such that

$$
\|x-z\|=\operatorname{dist}(x, \operatorname{co} \mathscr{U}(y)) \text {. }
$$

Proof. Let $z \in \operatorname{co} \mathscr{U}(y)$ and $\alpha=(x \eta \mid \eta)$ where $\eta \in \mathscr{H},\|\eta\|=1$. Since $\overline{W(z)} \subseteq \operatorname{co} \sigma(y)$ follows as in the proof (ii) $\Rightarrow$ (iii) of Theorem 4.1 , we have

$$
\|x-z\| \geq|((x-z) \eta \mid \eta)| \geq \operatorname{dist}(\alpha, \operatorname{co} \sigma(y)) .
$$

Since $\sigma(x) \subseteq \overline{W(x)}$, this implies

$$
\operatorname{dist}(x, \operatorname{co} \mathscr{U}(y)) \geq \sup _{\alpha \in \sigma(x)} \operatorname{dist}(\alpha, \operatorname{co} \sigma(y)) .
$$

Given $\alpha \in \mathbf{C}$, let $f(\alpha) \in \operatorname{co} \sigma(y)$ be such that $|\alpha-f(\alpha)|=\operatorname{dist}(\alpha, \operatorname{co} \sigma(y))$. Then $f$ is a continuous function on $\mathbf{C}$. Now define $z=f(x)$ through the spectral decomposition of $x$. Then

$$
\|x-z\|=\sup _{\alpha \in \sigma(x)}|\alpha-f(\alpha)|=\sup _{\alpha \in \sigma(x)} \operatorname{dist}(\alpha, \operatorname{co} \sigma(y)) .
$$

Moreover $\sigma(z)=f(\sigma(x)) \subseteq \operatorname{co} \sigma(y)$. Hence $z \in \overline{\operatorname{co}} \mathscr{U}(y)$ by Theorem 4.1.

Let $\mathscr{M}_{*, \text { sa }}$ (resp. $\mathscr{M}_{*,+}$ ) denote the set of all selfadjoint (resp. positive) elements in $\mathscr{M}_{*}$. The support projection of $\varphi \in \mathscr{M}_{*,+}$ is denoted by $s(\varphi)$. The Ky Fan functional of $\varphi \in \mathscr{M}_{*}$ is defined by

$$
K(\varphi, x)=\sup _{u \in \mathscr{U}} \operatorname{Re} \varphi\left(u^{*} x u\right), \quad x \in \mathscr{M} .
$$

It is known [1, p. 64] that if $\varphi, \psi \in \mathscr{M}_{*,+}$ and $\varphi(1)=\psi(1)$, then $\varphi \in \overline{\operatorname{co}} \mathscr{U}(\psi)$ if and only if $K(\varphi, q) \leq K(\psi, q)$ for every projection $q \in \mathscr{M}$. When $\mathscr{M}$ is a type III factor, it follows that $K(\varphi, q)=\varphi(1)$ for every $\varphi \in \mathscr{M}_{*,+}$ and every nonzero projection $q \in \mathscr{M}$. Hence the next lemma follows.

Lemma 4.3. Assume that $\mathscr{M}$ is a factor of type III. If $\varphi, \psi \in \mathscr{M}_{*,+}$ and $\varphi(1)=$ $\psi(1)$, then $\varphi \in \overline{\mathrm{co}} \mathscr{U}(\psi)$.

The next lemma is similar to Lemma 3.1(3).

Lemma 4.4. Assume that $\mathscr{M}$ is a factor of type III. If $\psi \in \mathscr{M}_{*, \text { sa }}$ and $\psi(1)=0$, then $0 \in \overline{\mathrm{co}} \mathscr{U}(\psi)$.

Proof. Let $\psi=\psi_{+}-\psi_{-}$be the Jordan decomposition of $\psi$. Suppose $\psi_{+}(1)=$ $\psi_{-}(1)>0$ and let $e=s\left(\psi_{+}\right)$. Then there exists a $u \in \mathscr{U}$ such that $u e u^{*}=$ $s\left(\psi_{-}\right)$and $u s\left(\psi_{-}\right) u^{*}=e$. Define $\omega=-u^{*} \psi u$. Since $s\left(\omega_{+}\right)=s\left(u^{*} \psi_{-} u\right)=e$, 
we have $\omega=e \omega e+e^{\perp} \omega e^{\perp}$ as well as $\psi=e \psi e+e^{\perp} \psi e^{\perp}$, and $\omega, \psi$ are positive on $\mathscr{M}_{e}$ and negative on $\mathscr{M}_{e^{\perp}}$. Furthermore it is easy to check that $\omega(e)=\psi(e)$ and $\omega\left(e^{\perp}\right)=\psi\left(e^{\perp}\right)$. Thus Lemma 4.3 implies $\omega \in \overline{\mathrm{co}} \mathscr{U}(\psi)$, and hence $0=\left(\psi+u \omega u^{*}\right) / 2 \in \overline{\mathrm{co}} \mathscr{U}(\psi)$.

Theorem 4.5. Assume that $\mathscr{M}$ is a factor of type III, and let $\varphi, \psi \in \mathscr{M}_{*, \mathrm{sa}}$. Then

$$
\operatorname{dist}(\varphi, \operatorname{co} \mathscr{U}(\psi))=\max \{\|\varphi\|-\|\psi\|,|\varphi(1)-\psi(1)|\},
$$

and there exists an $\omega \in \overline{\operatorname{co}} \mathscr{U}(\psi)$ such that $\|\varphi-\omega\|=\operatorname{dist}(\varphi, \operatorname{co} \mathscr{U}(\psi))$. In particular, $\varphi \in \overline{\mathrm{co}} \mathscr{U}(\psi)$ if and only if $\|\varphi\| \leq\|\psi\|$ and $\varphi(1)=\psi(1)$.

Proof. First let us prove the last part. If $\varphi \in \overline{\operatorname{co}} \mathscr{U}(\psi)$, then $\|\varphi\| \leq\|\psi\|$ and $\varphi(1)=\psi(1)$ are obvious. Conversely suppose $\|\varphi\| \leq\|\psi\|$ and $\varphi(1)=\psi(1)$. Then $\varphi_{+}(1) \leq \psi_{+}(1), \varphi_{-}(1) \leq \psi_{-}(1)$, and $\psi_{+}(1)-\varphi_{+}(1)=\psi_{-}(1)-\varphi_{-}(1)$. We can choose projections $q_{1}, q_{2} \in \mathscr{M}$ such that

$$
\begin{array}{ll}
q_{1} \leq s\left(\psi_{+}\right), & \psi_{+}\left(q_{1}\right)=\psi_{+}(1)-\varphi_{+}(1), \\
q_{2} \leq s\left(\psi_{-}\right), & \psi_{-}\left(q_{2}\right)=\psi_{-}(1)-\varphi_{-}(1) .
\end{array}
$$

Let $e=s\left(\psi_{+}\right)$and $q=\left(q_{1}+q_{2}\right)^{\perp}$. Define

$$
\tilde{\psi}=q_{1} \psi q_{1}+q_{2} \psi q_{2}+q \psi q .
$$

Then $\tilde{\psi}=e \tilde{\psi} e+e^{\perp} \tilde{\psi} e^{\perp}$, and $\tilde{\psi}, \psi$ are positive on $\mathscr{M}_{e}$ with $\tilde{\psi}(e)=\psi(e)$ and negative on $\mathscr{M}_{e^{\perp}}$ with $\tilde{\psi}\left(e^{\perp}\right)=\psi\left(e^{\perp}\right)$. Hence $\tilde{\psi} \in \overline{\operatorname{co}} \mathscr{U}(\psi)$ by Lemma 4.3. Also $\tilde{\psi}=q^{\perp} \tilde{\psi} q^{\perp}+q \tilde{\psi} q$ and $\tilde{\psi}\left(q^{\perp}\right)=\psi_{+}\left(q_{1}\right)-\psi_{-}\left(q_{2}\right)=0$. Hence $q \tilde{\psi} q \epsilon$ $\overline{\mathrm{co}} \mathscr{U}(\tilde{\psi})$ by Lemma 4.4. Now let $\omega=q \tilde{\psi} q$ which is in $\overline{\mathrm{co}} \mathscr{U}(\psi)$. So it suffices to show $\varphi \in \overline{\mathrm{co}} \mathscr{U}(\omega)$. We get

$$
\begin{aligned}
& \omega_{+}(1)=\tilde{\psi}_{+}(q)=\psi_{+}\left(q_{1}^{\perp}\right)=\varphi_{+}(1), \\
& \omega_{-}(1)=\tilde{\psi}_{-}(q)=\psi_{-}\left(q_{2}^{\perp}\right)=\varphi_{-}(1) .
\end{aligned}
$$

If either $\varphi_{+}(1)=0$ or $\varphi_{-}(1)=0$, then Lemma 4.3 implies $\varphi \in \overline{\operatorname{co}} \mathscr{U}(\omega)$. On the other hand, if $\varphi_{+}(1)>0$ and $\varphi_{-}(1)>0$, then $s\left(\left(u^{*} \omega u\right)_{+}\right)=s\left(\varphi_{+}\right)$for some $u \in \mathscr{U}$, and hence Lemma 4.3 implies $\varphi \in \overline{\operatorname{co}} \mathscr{U}\left(u^{*} \omega u\right)=\overline{\operatorname{co}} \mathscr{U}(\omega)$. Thus the last part is proved.

Next let us prove the main part. For every $\omega \in \operatorname{co} \mathscr{U}(\psi)$, we have

$$
\begin{aligned}
\|\varphi-\omega\| & \geq|\varphi(1)-\omega(1)|=|\varphi(1)-\psi(1)|, \\
\|\varphi-\omega\| & \geq\left|\varphi\left(p-p^{\perp}\right)-\omega\left(p-p^{\perp}\right)\right|=\left|\|\varphi\|-\omega\left(p-p^{\perp}\right)\right| \\
& \geq\|\varphi\|-\|\omega\| \geq\|\varphi\|-\|\psi\|
\end{aligned}
$$

where $p=s\left(\varphi_{+}\right)$. Hence

$$
\operatorname{dist}(\varphi, \operatorname{co} \mathscr{U}(\psi)) \geq \max \{\|\varphi\|-\|\psi\|,|\varphi(1)-\psi(1)|\} .
$$

The rest of the proof is divided into two cases. 
Case 1. Suppose $\varphi_{+}(1) \leq \psi_{+}(1)$ and $\varphi_{-}(1) \leq \psi_{-}(1)$. Considering $-\varphi,-\psi$ if necessary, we may suppose $\varphi(1) \leq \psi(1)$. First when $\varphi_{+}(1)>0$, define

$$
\omega=\left(1+\frac{\psi(1)-\varphi(1)}{\varphi_{+}(1)}\right) \varphi_{+}-\varphi_{-} .
$$

Then $\omega(1)=\varphi(1)$ and

$$
\begin{aligned}
\|\omega\| & =\varphi_{+}(1)+\psi(1)-\varphi(1)+\varphi_{-}(1)=\psi(1)+2 \varphi_{-} \\
& \leq \psi(1)+2 \psi_{-}(1)=\|\psi\|,
\end{aligned}
$$

so that $\omega \in \overline{\mathrm{co}} \mathscr{U}(\psi)$ by the last part already shown. Moreover $\|\varphi-\omega\|=\psi(1)-$ $\varphi(1)$. Second when $\varphi_{+}(1)=0$ and $\varphi_{-}(1)>0$, define $\omega=\left(\psi(1) / \varphi_{-}(1)\right) \varphi_{-}$. Then $\|\varphi-\omega\|=|\varphi(1)-\psi(1)|, \omega(1)=\psi(1)$ and $\|\omega\|=|\psi(1)| \leq\|\psi\|$; hence $\omega \in \overline{\mathrm{co}} \mathscr{U}(\psi)$. Third when $\varphi=0, \omega=\psi$ is enough.

Case 2. We may suppose $\varphi_{+}(1)>\psi_{+}(1)$ in the case remaining. First when $\varphi_{-}(1)>0$, define

$$
\omega=\frac{\psi_{+}(1)}{\varphi_{+}(1)} \varphi_{+}-\frac{\psi_{-}(1)}{\varphi_{-}(1)} \varphi_{-} .
$$

Then $\omega(1)=\psi(1)$ and $\|\omega\|=\|\psi\|$. Hence $\omega \in \overline{\operatorname{co}} \mathscr{U}(\psi)$ by the last part. Moreover

$$
\|\varphi-\omega\|=\left|\varphi_{+}(1)-\psi_{+}(1)\right|+\left|\varphi_{-}(1)-\psi_{-}(1)\right|,
$$

which is equal to $\|\varphi\|-\|\psi\|$ if $\varphi_{-}(1) \geq \psi_{-}(1)$, or to $|\varphi(1)-\psi(1)|$ if $\varphi_{-}(1)<$ $\psi_{-}(1)$. Second when $\varphi_{-}(1)=0$, define $\omega=\left(\psi(1) / \varphi_{+}(1)\right) \varphi_{+}$. Then $\|\varphi-\omega\|=$ $|\varphi(1)-\psi(1)|$ and $\omega \in \overline{\operatorname{co}} \mathscr{U}(\psi)$ as in Case 1.

The formulas in Theorems 4.2 and 4.5 are much simpler than those given in the previous sections. This means that the convex hulls of unitary orbits are very large in type III factors.

In the rest of this section, we estimate $\operatorname{dist}_{L^{p}}(x, \operatorname{co} \mathscr{U}(y))$ for selfadjoint elements $x, y$ in the Haagerup $L^{p}$-space [15] in case of type III $_{1}$ factors. To fix notations, we here give a very brief survey on Haagerup $L^{p}$-spaces (see [39] for details). Let $\mathscr{M}$ be an arbitrary von Neumann algebra. Let $\mathscr{N}$ be the crossed product of $\mathscr{M}$ by the modular automorphism group, which admits the faithful normal semifinite trace $\tau$ and the dual action $\theta$ satisfying $\tau \circ \theta_{s}=$ $e^{-s} \tau, s \in \mathbf{R}$. For $1 \leq p<\infty$, the Haagerup $L^{p}$-space $L^{p}(\mathscr{M})$ is defined by

$$
L^{p}(\mathscr{M})=\left\{x \in \widetilde{\mathcal{N}}: \theta_{s}(x)=e^{-s / p} x, s \in \mathbf{R}\right\} .
$$

Let $L^{p}(\mathscr{M})_{\text {sa }}\left(\right.$ resp. $\left.L^{p}(\mathscr{M})_{+}\right)$denote $L^{p}(\mathscr{M}) \cap \widetilde{\mathcal{N}_{\text {sa }}}\left(\right.$ resp. $\left.L^{p}(\mathscr{M}) \cap \widetilde{\mathcal{N}}_{+}\right)$. Then $L^{1}(\mathscr{M})$ is canonically order-isomorphic to $\mathscr{M}_{*}$ by a linear bijection $\varphi \in \mathscr{M}_{*} \mapsto$ $h_{\varphi} \in L^{1}(\mathscr{M})$, and the positive linear functional tr on $L^{1}(\mathscr{M})$ is defined by $\operatorname{tr}\left(h_{\varphi}\right)=\varphi(1), \varphi \in \mathscr{M}_{*}$. For $1 \leq p<\infty, L^{p}(\mathscr{M})$ is a Banach space with the norm $\|x\|_{p}=\operatorname{tr}\left(|x|^{p}\right)^{1 / p}$ and has all the expected properties, e.g. duality, Hölder's inequality, and uniform convexity in case of $1<p<\infty$. The unitary 
orbit of $y \in L^{p}(\mathscr{M})$ is given by $\mathscr{U}(y)=\left\{u y u^{*}: u \in \mathscr{U}\right\}$ where $\mathscr{U}$ is the unitaries in $\mathscr{M}$. If $y \in L^{p}(\mathscr{M})$ and $u \in \mathscr{U}$, then $\left\|u y u^{*}\right\|_{p}=\|y\|_{p}$. If $y \in$ $L^{p}(\mathscr{M})_{+}$, then the support projection $s(y) \quad\left(=e_{(0, \infty)}(y)\right)$ of $y$ is in $\mathscr{M}$. Note that when $\mathscr{U}$ is semifinite, the Haagerup $L^{p}$-space coincides with $L^{p}(\mathscr{M})$ in the sense of $\S 1$.

Lemma 4.6. If $y \in L^{p}(\mathscr{U})_{\mathrm{sa}}, 1<p<\infty$, and $x \in \overline{\mathrm{co}}^{L^{p}} \mathscr{U}(y)$, then $\left\|x_{+}\right\|_{p} \leq$ $\left\|y_{+}\right\|_{p}$ and $\left\|x_{-}\right\|_{p} \leq\left\|y_{-}\right\|_{p}$.

Proof. By [25, Theorem 4.4] (also [21, Lemma 5.1]), we may suppose $x \in$ co $\mathscr{U}(y)$, so $x=\sum_{i=1}^{n} \lambda_{i} u_{i} y u_{i}^{*}$ with $u_{i} \in \mathscr{U}, \lambda_{i}>0$ and $\sum_{i=1}^{n} \lambda_{i}=1$. Let $1 / p+1 / q=1, z_{1}=\sum_{i=1}^{n} \lambda_{i} u_{i} y_{+} u_{i}^{*}$ and $z_{2}=\sum_{i=1}^{n} \lambda_{i} u_{i} y_{-} u_{i}^{*}$. Then $x=$ $z_{1}-z_{2}$. By [39, Proposition II.33] and Hölder's inequality, we get

$$
\begin{aligned}
\left\|x_{+}\right\|_{p}^{p} & =\operatorname{tr}\left(x x_{+}^{p-1}\right)=\operatorname{tr}\left(z_{1} x_{+}^{p-1}\right)-\operatorname{tr}\left(z_{2} x_{+}^{p-1}\right) \\
& \leq \operatorname{tr}\left(z_{1} x_{+}^{p-1}\right) \leq\left\|z_{1}\right\|_{p}\left\|x_{+}^{p-1}\right\|_{q} \leq\left\|y_{+}\right\|_{p}\left\|x_{+}\right\|_{p}^{p / q},
\end{aligned}
$$

so that $\left\|x_{+}\right\|_{p} \leq\left\|y_{+}\right\|_{p}$, and similarly $\left\|x_{-}\right\|_{p} \leq\left\|y_{-}\right\|_{p}$.

Lemma 4.7. Assume that $\mathscr{M}$ is a factor of type III, and let $e \in \mathscr{M}$ be a projection. If $y \in L^{p}(\mathscr{M})_{+}, 1<p<\infty$, and $s(y) \leq e$, then $0 \in \overline{\operatorname{co}}^{L^{p}} \mathscr{U}_{e}(y)$.

Proof. By approximation, we may assume $s(y) \neq e$. Then for each $n \geq 1$, there are $u_{1}, \ldots, u_{n} \in \mathscr{U}_{e}$ such that $u_{i} s(y) u_{i}^{*}, 1 \leq i \leq n$, are mutually orthogonal. Let $z=n^{-1} \sum_{i=1}^{n} u_{i} y u_{i}^{*}$. Then

$$
\|z\|_{p}^{p}=n^{-p} \operatorname{tr}\left(\sum_{i=1}^{n}\left(u_{i} y u_{i}^{*}\right)^{p}\right)=n^{1-p}\|y\|_{p}^{p} .
$$

This shows the lemma.

Theorem 4.8. Assume that $\mathscr{M}$ is a factor of type $\mathrm{III}_{1}$, and let $x, y \in L^{p}(\mathscr{M})_{\text {sa }}$ with $1<p<\infty$. Then

$$
\operatorname{dist}_{L^{p}}(x, \operatorname{co} \mathscr{U}(y))=\left\{\left(\left\|x_{+}\right\|_{p}-\left\|y_{+}\right\|_{p}\right)_{+}^{p}+\left(\left\|x_{-}\right\|_{p}-\left\|y_{-}\right\|_{p}\right)_{+}^{p}\right\}^{1 / p} .
$$

In particular, $x \in \overline{\mathrm{co}}^{L^{\prime \prime}} \mathscr{U}(y)$ if and only if $\left\|x_{+}\right\|_{p} \leq\left\|y_{+}\right\|_{p}$ and $\left\|x_{-}\right\|_{p} \leq\left\|y_{-}\right\|_{p}$. Proof. First let us prove the last part. Suppose $\left\|x_{+}\right\|_{p} \leq\left\|y_{+}\right\|_{p}$ and $\left\|x_{-}\right\|_{p} \leq$ $\left\|y_{-}\right\|_{p}$. Let $e=s\left(y_{+}\right)$. By Lemma 4.7, there exist $z_{1} \in \overline{\operatorname{co}}^{p} \mathscr{U}_{e}\left(y_{+}\right)$and $z_{2} \in \overline{\mathrm{co}}^{L^{p}} \mathscr{U}_{e^{\perp}}\left(y_{-}\right)$such that $\left\|z_{1}\right\|_{p}=\left\|x_{+}\right\|_{p}$ and $\left\|z_{2}\right\|_{p}=\left\|x_{-}\right\|_{p}$. Letting $z=$ $z_{1}-z_{2}$, we get $z_{+}=z_{1}, z_{-}=z_{2}$, and $z \in \overline{\mathrm{co}}^{L^{p}} \mathscr{U}(y)$. Then [21, Theorem 5.3] implies that $x$ is in the $\|\cdot\|_{p}$-closure of $\mathscr{U}(z)$ and hence $x \in \overline{\mathrm{co}}^{L^{p}} \mathscr{U}(y)$. The converse is Lemma 4.6. 
Next let us prove the main part. For every $z \in \operatorname{co} \mathscr{U}(y)$, by [21, Lemma 5.1] and Lemma 4.6 we have

$$
\begin{aligned}
\|x-z\|_{p}^{p} & \geq\left|\left\|x_{+}\right\|_{p}-\left\|z_{+}\right\|_{p}\right|^{p}+\left|\left\|x_{-}\right\|_{p}-\left\|z_{-}\right\|_{p}\right|^{p} \\
& \geq\left(\left\|x_{+}\right\|_{p}-\left\|z_{+}\right\|_{p}\right)_{+}^{p}+\left(\left\|x_{-}\right\|_{p}-\left\|z_{-}\right\|_{p}\right)_{+}^{p} \\
& \geq\left(\left\|x_{+}\right\|_{p}-\left\|y_{+}\right\|_{p}\right)_{+}^{p}+\left(\left\|x_{-}\right\|_{p}-\left\|y_{-}\right\|_{p}\right)_{+}^{p} .
\end{aligned}
$$

Hence

$$
\operatorname{dist}_{L^{p}}(x, \operatorname{co} \mathscr{U}(y)) \geq\left\{\left(\left\|x_{+}\right\|_{p}-\left\|y_{+}\right\|_{p}\right)_{+}^{p}+\left(\left\|x_{-}\right\|_{p}-\left\|y_{-}\right\|_{p}\right)_{+}^{p}\right\}^{1 / p} .
$$

Here it is worth noting that the above inequality is valid when $\mathscr{M}$ is a general von Neumann algebra. Conversely define

$$
z=\frac{\left\|x_{+}\right\|_{p} \wedge\left\|y_{+}\right\|_{p}}{\left\|x_{+}\right\|_{p}} x_{+}-\frac{\left\|x_{-}\right\|_{p} \wedge\left\|y_{-}\right\|_{p}}{\left\|x_{-}\right\|_{p}} x_{-},
$$

where the first (resp. second) term of the right-hand side expression is meant to be 0 if $x_{+}=0$ (resp. $x_{-}=0$ ). Since $\left\|z_{+}\right\|_{p} \leq\left\|y_{+}\right\|_{p}$ and $\left\|z_{-}\right\|_{p} \leq\left\|y_{-}\right\|_{p}$, we get $z \in \overline{\mathrm{co}}^{L^{p}} \mathscr{U}(y)$ by the last part previously shown. Furthermore

$$
\|x-z\|_{p}^{p}=\left(\left\|x_{+}\right\|_{p}-\left\|y_{+}\right\|_{p}\right)_{+}^{p}+\left(\left\|x_{-}\right\|_{p}-\left\|y_{-}\right\|_{p}\right)_{+}^{p} .
$$

In Theorem 4.8 , because of uniform convexity of $L^{p}(\mathscr{M})$, there exists a unique $z \in \overline{\mathrm{co}}^{L^{p}} \mathscr{U}(y)$ such that $\|x-z\|=\operatorname{dist}_{L^{p}}(x, \operatorname{co} \mathscr{U}(y))$. Theorem 4.8 is due to homogeneity of the normal state space of type III $_{1}$ factors [8]. Indeed the next theorem shows that it does not hold unless $\mathscr{M}$ is a type III $_{1}$ factor.

Theorem 4.9. Let $1<p<\infty$ and suppose that $x \in \overline{\mathrm{co}}^{L^{p}} \mathscr{U}(y)$ for every $x$, $y \in L^{p}(\mathscr{M})_{+}$with $\|x\|_{p}=\|y\|_{p}=1$. Then $\mathscr{M}$ is a factor of type $\mathrm{III}_{1}$.

Proof. For $1 \leq q<\infty$, consider the following condition:

$\left(\mathrm{H}_{q}\right) \quad x$ is in the $\|\cdot\|_{q}$-closure of $\mathscr{U}(y)$ for every $x, y \in L^{q}(\mathscr{M})_{+}$with $\|x\|_{q}=\|y\|_{q}=1$.

Connes and Størmer [8] proved that $\left(\mathrm{H}_{1}\right)$ holds if and only if $\mathscr{M}$ is a factor of type III $_{1}$. Noting that $\left\|u y u^{*}\right\|_{p}=\|y\|_{p}$ for all $u \in \mathscr{U}$, we see by uniform convexity that the assumption in the theorem implies $\left(\mathrm{H}_{p}\right)$. So it suffices to show that $\left(\mathrm{H}_{p}\right) \Rightarrow\left(\mathrm{H}_{1}\right)$. If $x, y \in L^{q}(\mathscr{M})_{+}$and $\|x\|_{q}=\|y\|_{q}=1$, then

$$
\begin{aligned}
\|x-y\|_{q} & =\frac{1}{2}\left\|\left(x^{1 / 2}+y^{1 / 2}\right)\left(x^{1 / 2}-y^{1 / 2}\right)+\left(x^{1 / 2}-y^{1 / 2}\right)\left(x^{1 / 2}+y^{1 / 2}\right)\right\|_{q} \\
& \leq\left\|\left(x^{1 / 2}+y^{1 / 2}\right)\left(x^{1 / 2}-y^{1 / 2}\right)\right\|_{q} \\
& \leq\left(\left\|x^{1 / 2}\right\|_{2 q}+\left\|y^{1 / 2}\right\|_{2 q}\right)\left\|x^{1 / 2}-y^{1 / 2}\right\|_{2 q} \\
& =2\left\|x^{1 / 2}-y^{1 / 2}\right\|_{2 q} .
\end{aligned}
$$

This shows that $\left(\mathrm{H}_{2 q}\right) \Rightarrow\left(\mathrm{H}_{q}\right)$. Furthermore, if $1 \leq q \leq q^{\prime}<\infty$, then $\left(\mathbf{H}_{q}\right) \Rightarrow\left(\mathbf{H}_{q^{\prime}}\right)$ follows from the generalized Powers-Størmer inequality proved by Kosaki (appendix of [21]). Thus we have the desired conclusion. 
For $1 \leq p<\infty$, let $L^{p}(\mathscr{M})_{+, 1} / \mathscr{U}$ denote the space of closed unitary orbits in $\left\{x \in L^{p}(\mathscr{M})_{+}:\|x\|_{p}=1\right\}$. The above argument shows that $L^{p}(\mathscr{M})_{+, 1} / \mathscr{U}$ is a singleton if and only if $\mathscr{M}$ is a type III $_{1}$ factor. The second-named author [29] recently obtained some results on diameters of $L^{p}(\mathscr{M})_{+, 1} / \mathscr{U}$ for factors of type III $_{\lambda}, 0<\lambda<1$, using the discrete Haagerup $L^{p}$-spaces (see [7] for the special case $p=1$ ).

\section{Distance BetWeEN CONVEX HUlls OF UNITARY ORBITS}

We denote by $\operatorname{dist}(\operatorname{co} \mathscr{U}(x)$, co $\mathscr{U}(y))$ the $\|\cdot\|$-distance between co $\mathscr{U}(x)$ and $\operatorname{co} \mathscr{U}(y)$ for $x, y \in \mathscr{M}$, and by $\operatorname{dist}(\operatorname{co} \mathscr{U}(\varphi), \operatorname{co} \mathscr{U}(\psi))$ the same for $\varphi, \psi \in$ $\mathscr{M}_{*}$. In this section, we establish the formulas of these distances for selfadjoint elements. These formulas are very simple compared with those established in the previous sections.

Theorem 5.1. (1) Assume that $\mathscr{M}$ is a finite factor with the normalized trace $\tau$. If $x, y \in \mathscr{M}$, then

$$
\operatorname{dist}(\operatorname{co} \mathscr{U}(x), \operatorname{co} \mathscr{U}(y))=|\tau(x)-\tau(y)| .
$$

(2) Assume that $\mathscr{M}$ is a $\sigma$-finite infinite semifinite factor. If $x, y \in \mathscr{M}_{\mathrm{sa}}$, then

$$
\operatorname{dist}(\operatorname{co} \mathscr{U}(x), \operatorname{co} \mathscr{U}(y))=\operatorname{dist}\left(\operatorname{co} \sigma_{e}(x), \operatorname{co} \sigma_{e}(y)\right) .
$$

(3) Assume that $\mathscr{M}$ is a $\sigma$-finite factor of type III. If $x, y \in \mathscr{M}$ are normal, then

$$
\operatorname{dist}(\operatorname{co} \mathscr{U}(x), \operatorname{co} \mathscr{U}(y))=\operatorname{dist}(\operatorname{co} \sigma(x), \operatorname{co} \sigma(y)) .
$$

Proof. (1) is immediate from the well-known fact that $\tau(x) 1 \in \overline{\mathrm{co}} \mathscr{U}(x)$ for all $x \in \mathscr{M}$. (More generally, when $\mathscr{M}$ is a finite von Neumann algebra, we have $\operatorname{dist}(\operatorname{co} \mathscr{U}(x), \operatorname{co} \mathscr{U}(y))=\left\|x^{\natural}-y^{\natural}\right\|$ for every $x, y \in \mathscr{M}$ where $x \mapsto x^{\natural}$ is the center-valued trace $[12, \S I I I .4]$.

(2) Let $x^{\prime} \in \operatorname{co} \mathscr{U}(x)$ and $y^{\prime} \in \operatorname{co} \mathscr{U}(y)$. Since Theorem 3.3 implies

$$
\begin{aligned}
\min \sigma_{e}(x) & \leq \min \sigma_{e}\left(x^{\prime}\right) \leq \max \sigma_{e}\left(x^{\prime}\right) \leq \max \sigma_{e}(x), \\
\min \sigma_{e}(y) & \leq \min \sigma_{e}\left(y^{\prime}\right) \leq \max \sigma_{e}\left(y^{\prime}\right) \leq \max \sigma_{e}(y),
\end{aligned}
$$

by Proposition 1.1(1) we have

$$
\left\|x^{\prime}-y^{\prime}\right\| \geq\left|\max \sigma_{e}\left(x^{\prime}\right)-\max \sigma_{e}\left(y^{\prime}\right)\right| \geq \operatorname{dist}\left(\operatorname{co} \sigma_{e}(x), \operatorname{co} \sigma_{e}(y)\right) .
$$

Conversely, if $\min \sigma_{e}(x) \leq \alpha \leq \max \sigma_{e}(x)$ and $\min \sigma_{e}(y) \leq \beta \leq \max \sigma_{e}(y)$, then $\alpha 1 \in \overline{\operatorname{co}} \mathscr{U}(x)$ and $\beta 1 \in \overline{\mathrm{co}} \mathscr{U}(y)$ by Theorem 3.3, and hence

$$
\operatorname{dist}(\operatorname{co} \mathscr{U}(x), \operatorname{co} \mathscr{U}(y)) \leq|\alpha-\beta| \text {. }
$$

(3) If $x^{\prime} \in \operatorname{co} \mathscr{U}(x)$ and $y^{\prime} \in \operatorname{co} \mathscr{U}(y)$, then we have

$$
\left\|x^{\prime}-y^{\prime}\right\| \geq \operatorname{dist}\left(\overline{W\left(x^{\prime}\right)}, \overline{W\left(y^{\prime}\right)}\right) \geq \operatorname{dist}(\operatorname{co} \sigma(x), \operatorname{co} \sigma(y)),
$$

since $\overline{W\left(x^{\prime}\right)} \subseteq \operatorname{co} \sigma(x)$ and $\overline{W\left(y^{\prime}\right)} \subseteq \operatorname{co} \sigma(y)$ (see the proof (ii) $\Rightarrow$ (iii) of Theorem 4.1). Conversely, if $\alpha \in \operatorname{co} \sigma(x)$ and $\beta \in \operatorname{co} \sigma(y)$, then $\alpha 1 \in \overline{\operatorname{co}} \mathscr{U}(x)$ and $\beta 1 \in \overline{\operatorname{co}} \mathscr{U}(y)$ by Theorem 4.1, and hence $\operatorname{dist}(\operatorname{co} \sigma(x), \operatorname{co} \sigma(y)) \leq|\alpha-\beta|$. 
Theorem 5.2. Assume that $\mathscr{M}$ is any factor. If $\varphi, \psi \in \mathscr{M}_{*, \mathrm{sa}}$, then

$$
\operatorname{dist}(\operatorname{co} \mathscr{U}(\varphi), \operatorname{co} \mathscr{U}(\psi))=|\varphi(1)-\psi(1)| \text {. }
$$

Proof. Let us prove $\operatorname{dist}(\operatorname{co} \mathscr{U}(\varphi), \operatorname{co} \mathscr{U}(\psi)) \leq|\varphi(1)-\psi(1)|$ in each type of $\mathscr{M}$ (the reversed inequality is obvious).

(1) Let $\mathscr{M}$ be a finite factor. It suffices to show that if $x, y \in L^{1}(\mathscr{M})_{\text {sa }}$, then

$$
\operatorname{dist}_{L^{1}}(\operatorname{co} \mathscr{U}(x), \operatorname{co} \mathscr{U}(y)) \leq|\tau(x)-\tau(y)| .
$$

But this follows from Theorem 2.3 together with

$$
(\tau(x) / \tau(1)) 1 \prec x, \quad(\tau(y) / \tau(1)) 1 \prec y .
$$

(2) Let $\mathscr{M}$ be an infinite semifinite factor. We need to show the above inequality in $(1)$ for every $x, y \in L^{1}(\mathscr{M})_{\text {sa }}$. Because this is obvious from Lemma 3.1(3) if $\tau(x)=0$ or $\tau(y)=0$, we may assume that $\tau(x)$ and $\tau(y)$ are nonzero. Let $\tilde{x}=x / \tau(x), \tilde{y}=y / \tau(y)$, and

$$
\alpha_{n}=\min \left\{\int_{0}^{n} \lambda_{t}(\tilde{x}) d t, \int_{0}^{n} \lambda_{t}(\tilde{y}) d t, 1\right\}, \quad n \geq 1 .
$$

Then $\alpha_{n} \uparrow 1$ since $\tau\left(\tilde{x}_{+}\right) \geq \tau(\tilde{x})=1$ and $\tau\left(\tilde{y}_{+}\right) \geq \tau(\tilde{y})=1$. Furthermore $\alpha_{n}-\alpha_{n-1} \downarrow 0$ where $\alpha_{0}=0$. Hence we can choose a $z \in L^{1}(\mathscr{M})_{+}$such that $\lambda_{t}(z)=\alpha_{n}-\alpha_{n-1}$ for $n-1 \leq t<n$. Since $z \prec \tilde{x}_{+}$and $\tau(z)=1=\tau(\tilde{x})$, we get $z \prec \tilde{x}$ and hence $\tau(x) z \prec x$. Similarly $\tau(y) z \prec y$. Thus Theorem 3.5(4) implies $\tau(x) z \in \overline{\mathrm{co}}^{L^{1}} \mathscr{U}(x)$ and $\tau(y) z \in \overline{\mathrm{co}}^{L^{1}} \mathscr{U}(y)$, so that we have the desired inequality.

(3) Let $\mathscr{M}$ be a type III factor. For any normal state $\omega$ on $\mathscr{M}$, by Theorem 4.1 we get $\varphi(1) \omega \in \overline{\mathrm{co}} \mathscr{U}(\varphi)$ and $\psi(1) \omega \in \overline{\mathrm{co}} \mathscr{U}(\psi)$, implying the desired inequality.

Indeed the above proofs have shown the following: In (1)-(3) of Theorem 5.1, there exist $x^{\prime} \in \overline{\operatorname{co}} \mathscr{U}(x)$ and $y^{\prime} \in \overline{\mathrm{co}} \mathscr{U}(y)$ such that $\left\|x^{\prime}-y^{\prime}\right\|=$ $\operatorname{dist}(\operatorname{co} \mathscr{U}(x), \operatorname{co} \mathscr{U}(y))$. In Theorem 5.2, there exist $\varphi^{\prime} \in \overline{\operatorname{co}} \mathscr{U}(\varphi)$ and $\psi^{\prime} \in$ $\overline{\operatorname{co}} \mathscr{U}(\psi)$ such that $\left\|\varphi^{\prime}-\psi^{\prime}\right\|=|\varphi(1)-\psi(1)|$.

\section{THE NONFACTOR CASE}

Let $\mathscr{M}$ be a von Neumann algebra on a separable Hilbert space $\mathscr{H}$. We decompose $\{\mathscr{M}, \mathscr{H}\}$ into factors as the direct integral:

$$
\{\mathscr{M}, \mathscr{H}\}=\int_{\Gamma}^{\oplus}\{\mathscr{M}(\gamma), \mathscr{H}(\gamma)\} d \nu(\gamma)
$$

where $\gamma \mapsto\{\mathscr{M}(\gamma), \mathscr{H}(\gamma)\}$ is a measurable field of factors on a standard $\sigma$-finite measure space $(\Gamma, \mathscr{B}, \nu)$, Given $x \in \mathscr{M}$ (resp. $\varphi \in \mathscr{M}_{*}$ ), there exists a unique (in the a.e. sense) measurable field $x(\cdot) \in \prod_{\gamma \in \Gamma} \mathscr{M}(\gamma)\left(\right.$ resp. $\left.\varphi(\cdot) \in \prod_{\gamma \in \Gamma} \mathscr{M}(\gamma)_{*}\right)$ 
such that

$$
\begin{array}{rlr}
x=\int_{\Gamma}^{\oplus} x(\gamma) d \nu(\gamma) & \left(\varphi=\int_{\Gamma}^{\oplus} \varphi(\gamma) d \nu(\gamma)\right), \\
\|x\|=\underset{\gamma \in \Gamma}{\operatorname{ess} \sup \|x(\gamma)\|} & \left(\|\varphi\|=\int_{\Gamma}\|\varphi(\gamma)\| d \nu(\gamma)\right) .
\end{array}
$$

If $x \in \mathscr{M}_{\text {sa }}$ (resp. $\varphi \in \mathscr{M}_{*, \text { sa }}$ ), then we can assume that $x(\gamma) \in \mathscr{M}(\gamma)_{\text {sa }}$ (resp. $\left.\varphi(\gamma) \in \mathscr{M}(\gamma)_{*, \text { sa }}\right)$ for all $\gamma \in \Gamma$. In the following, we always keep the above correspondence between $x$ and $x(\cdot)$, or between $\varphi$ and $\varphi(\cdot)$. For more on theory of direct integral decompositions, we refer to [12, Chapter II] and [38, §IV.8].

Together with the estimates established in $\S \S 2-5$, the next theorems give the complete estimates of distances for convex hulls of unitary orbits.

Theorem 6.1. If $x, y \in \mathscr{M}_{\mathrm{sa}}$, then

$$
\operatorname{dist}(x, \operatorname{co} \mathscr{U}(y))=\underset{\gamma \in \Gamma}{\operatorname{ess} \sup } \operatorname{dist}(x(\gamma), \operatorname{co} \mathscr{U}(y(\gamma))),
$$

where $\mathscr{U}(y(\gamma))$ is the unitary orbit of $y(\gamma)$ in $\mathscr{M}(\gamma)$, and there exists a $z \in$ $\overline{\mathrm{co}} \mathscr{U}(y)$ such that $\|x-z\|=\operatorname{dist}(x, \operatorname{co} \mathscr{U}(y))$.

Theorem 6.2. If $\varphi, \psi \in \mathscr{M}_{*, \mathrm{sa}}$, then

$$
\operatorname{dist}(\varphi, \operatorname{co} \mathscr{U}(\psi))=\int_{\Gamma} \operatorname{dist}(\varphi(\gamma), \operatorname{co} \mathscr{U}(\psi(\gamma))) d \nu(\gamma),
$$

where $\mathscr{U}(\psi(\gamma))$ is the unitary orbit of $\psi(\gamma)$ in $\mathscr{M}(\gamma)_{*}$, and there exists an $\omega \in \overline{\operatorname{co}} \mathscr{U}(\psi)$ such that $\|\varphi-\omega\|=\operatorname{dist}(\varphi, \operatorname{co} \mathscr{U}(\psi))$.

Theorem 6.3. (1) If $x, y \in \mathscr{M}_{\mathrm{sa}}$, then

$$
\operatorname{dist}(\operatorname{co} \mathscr{U}(x), \operatorname{co} \mathscr{U}(y))=\underset{\gamma \in \Gamma}{\operatorname{ess} \sup } \operatorname{dist}(\operatorname{co} \mathscr{U}(x(\gamma)), \operatorname{co} \mathscr{U}(y(\gamma))) .
$$

(2) If $\varphi, \psi \in \mathscr{M}_{*, \mathrm{sa}}$, then

$$
\begin{aligned}
\operatorname{dist}(\operatorname{co} \mathscr{U}(\varphi), \operatorname{co} \mathscr{U}(\psi)) & =\int_{\Gamma} \operatorname{dist}(\operatorname{co} \mathscr{U}(\varphi(\gamma)), \operatorname{co} \mathscr{U}(\psi(\gamma))) d \nu(\gamma) \\
& =\int_{\Gamma}|\varphi(\gamma)(1)-\psi(\gamma)(1)| d \nu(\gamma) .
\end{aligned}
$$

Here we only give a sketch of the proof of Theorem 6.1. Taking the $\nu$ completion of $\mathscr{B}$, we may assume that $\mathscr{B}$ is complete with respect to $\nu$. Let $F(\gamma)=\overline{\mathrm{co}} \mathscr{U}(y(\gamma))$ and $f(\gamma)=\operatorname{dist}(x(\gamma)$, co $\mathscr{U}(y(\gamma)))$ for $\gamma \in \Gamma$. By $\left[19\right.$, Lemma 3.1], there is a sequence $\left\{z_{j}(\cdot)\right\}$ of measurable fields such that $\left\{z_{j}(\gamma)\right\}$ is weakly dense in $F(\gamma)$ for every $\gamma \in \Gamma$. Also there is a sequence $\left\{\varphi_{n}(\cdot)\right\}$ of measurable fields in $\prod_{\gamma \in \Gamma} \mathscr{M}(\gamma)_{*}$ such that $\left\{\varphi_{n}(\cdot)\right\}$ is norm-dense in the unit sphere of $\mathscr{M}(\gamma)_{*}$ for every $\gamma \in \Gamma$. Since Theorems $2.1,3.3$ and 4.1 imply that $F(\gamma)=\overline{\mathrm{co}}^{\mathrm{w}} \mathscr{U}(y(\gamma))$ is weakly compact, by the minimax theorem 
[14, Theorem 2] we have

$$
\begin{aligned}
f(\gamma) & =\inf _{a \in F(\gamma)} \sup _{\substack{\omega \in \mathscr{K}(\gamma)_{*} \\
\|\omega\| \leq 1}} \operatorname{Re}\langle x(\gamma)-a, \omega\rangle \\
& =\sup _{n \geq 1} \inf _{j \geq 1} \operatorname{Re}\left\langle x(\gamma)-z_{j}(\gamma), \varphi_{n}(\gamma)\right\rangle, \quad \gamma \in \Gamma,
\end{aligned}
$$

showing measurability of $f$. By the measurable selection method $[4,22]$ together with Theorems 2.4, 3.6 and 4.2, we can choose a measurable field $z(\cdot)$ such that $z(\gamma) \in F(\gamma)$ and $\|x(\gamma)-z(\gamma)\|=f(\gamma)$ for all $\gamma \in \Gamma$. It remains to prove $z \in \overline{c o} \mathscr{U}(y)$. To do so, it suffices to show that given $\varepsilon>0$ there exist measurable fields $u_{1}(\cdot), \ldots, u_{N}(\cdot)$ of unitaries and measurable functions $f_{1}, \ldots, f_{N} \geq 0$ on $\Gamma$ with $\sum_{i=1}^{N} f_{i}(\gamma)=1$ satisfying

$$
\left\|x(\gamma)-\sum_{i=1}^{N} f_{i}(\gamma) u_{i}(\gamma) y(\gamma) u_{i}(\gamma)^{*}\right\| \leq \varepsilon, \quad \gamma \in \Gamma .
$$

When $\mathscr{M}(\gamma)$ is finite (resp. infinite semifinite) for every $\gamma \in \Gamma$, we can show this by tracing the proof (iii) $\Rightarrow$ (i) of Theorem 2.1 (resp. (iv) $\Rightarrow$ (i) of Theorem 3.3) with additional parameter $\gamma \in \Gamma$. Here, by [19, Lemma 2.2] and the measurable selection method, all scalars and operators can be replaced by corresponding measurable functions and measurable fields of operators, while we omit the technical details. Also when $\mathscr{M}(\gamma)$ is of type III for every $\gamma \in \Gamma$, it is not difficult to show the above assertion. Combining those cases, we get $z \in \overline{\mathrm{co}} \mathscr{U}(y)$, and Theorem 6.1 is proved. Theorems 6.2 and 6.3 are proved in similar ways. The detailed proofs of the theorems are left to the reader.

When $\mathscr{M}$ is acting on a separable Hilbert space, it is easy to see that for each $x, y \in \mathscr{M}, x \in \overline{\operatorname{co}}^{\mathrm{w}} \mathscr{U}(y)$ if and only if $x(\gamma) \in \overline{\mathrm{co}}^{\mathrm{w}} \mathscr{U}(y(\gamma))$ a.e. Hence the above argument shows that if $y \in \mathscr{M}_{\text {sa }}$ and $x \in \overline{\mathrm{co}}^{\mathrm{w}} \mathscr{U}(y)$, then $x \in \overline{\mathrm{co}} \mathscr{U}(y)$. Furthermore for each $x, y \in \mathscr{M}$ when $\mathscr{M}$ is $\sigma$-finite, if $x \in \overline{c o}^{\mathrm{w}} \mathscr{U}(y)$, then there exists a countably generated von Neumann subalgebra $\mathscr{M}_{0}$ of $\mathscr{M}$ such that $y \in \mathscr{M}_{0}$ and $x \in \overline{\mathrm{co}}^{\mathrm{w}}\left\{u y u^{*}: u \in \mathscr{M}_{0}\right.$ unitary $\}$. Thus we obtain the following theorem.

Theorem 6.4. Assume that $\mathscr{M}$ is a $\sigma$-finite von Neumann algebra. Then the equality $\overline{\mathrm{co}} \mathscr{U}(y)=\overline{\mathrm{co}}^{\mathrm{w}} \mathscr{U}(y)$ holds for every $y \in \mathscr{M}_{\mathrm{sa}}$.

It is known [17, Corollary 4.17] (also [34, Theorem 3.7]) that if $\mathscr{M}$ is $\sigma$ finite, then $\overline{\mathrm{co}} \mathscr{U}(y) \cap \mathscr{Z}=\overline{\mathrm{co}}^{\mathrm{w}} \mathscr{U}(y) \cap \mathscr{Z}$ for every $y \in \mathscr{M}$ where $\mathscr{Z}$ is the center of $\mathscr{M}$. An interesting problem is to distinguish $y \in \mathscr{M}$ for which the equality $\overline{\mathrm{co}} \mathscr{U}(y)=\overline{\mathrm{co}}^{\mathrm{w}} \mathscr{U}(y)$ holds. But it should be pointed out that we have

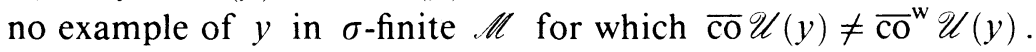

\section{REFERENCES}

1. P. M. Alberti and A. Uhlmann, Stochasticity and partial order, VEB Deutscher Verlag Wiss., Berlin, 1982.

2. T. Ando, Majorization, doubly stochastic matrices and comparison of eigenvalues, Lecture Notes, Hokkaido Univ., Sapporo, 1982; Linear Algebra Appl. 118 (1989), 163-248. 
3. G. Birkhoff, Tres observaciones sobre el algebra lineal, Univ. Nac. Tucuman Rev. Ser. A 5 (1946), 147-151.

4. C. Castaing and M. Valadier, Convex analysis and measurable multifunctions, Lecture Notes in Math., vol. 580, Springer-Verlag, 1977.

5. K. M. Chong, Some extensions of a theorem of Hardy, Littlewood and Pólya and their applications, Canad. J. Math. 26 (1974), 1321-1340.

6. _ Doubly stochastic operators and rearrangement theorems, J. Math. Anal. Appl. 56 (1976), 309-316.

7. A. Connes, U. Haagerup and E. Størmer, Diameters of state spaces of type III factors, Operator Algebras and their Connections with Topology and Ergodic Theory $(\mathrm{H}$. Araki et al., eds.), Lecture Notes in Math., vol. 1132, Springer-Verlag, 1985, pp. 91-116.

8. A. Connes and E. Størmer, Homogeneity of the state space of factors of type III $_{1}$, J. Funct. Anal. 28 (1978), 187-196.

9. J. B. Conway, The numerical range and a certain convex set in an infinite factor, J. Funct. Anal. 5 (1970), 428-435.

10. J. Dixmier, Les anneaux d'opérateurs de classe finie, Ann. Sci. École Norm. Sup. 66 (1949), 209-261.

11. __ Formes linéaires sur un anneau d'opérateurs, Bull. Soc. Math. France 81 (1953), 9-39.

12. __ Les algèbres d'opérateurs dans l'espace Hilbertien (algèbres de von Neumann), 2nd ed., Gauthier-Villars, Paris, 1969.

13. T. Fack and H. Kosaki, Generalized s-numbers of $\tau$-measurable operators, Pacific J. Math. 123 (1986), 269-300.

14. K. Fan, Minimax theorems, Proc. Nat. Acad. Sci. U.S.A. 39 (1953), 42-47.

15. U. Haagerup, $L^{p}$-spaces associated with an arbitrary von Neumann algebra, Colloq. Internat. CNRS, no. 274, CNRS, Paris, 1979, pp. 175-184.

16. P. R. Halmos, A Hilbert space problem book, Springer-Verlag, 1974.

17. H. Halpern, Essential central spectrum and range for elements of a von Neumann algebra, Pacific J. Math. 43 (1972), 349-380.

18. F. Hiai, Majorization and stochastic maps in von Neumann algebras, J. Math. Anal. Appl. 127 (1987), 18-48.

19. __ Spectral relations and unitary mixing in semifinite von Neumann algebras, Hokkaido Math. J. 17 (1988), 117-137.

20. F. Hiai and Y. Nakamura, Majorizations for generalized s-numbers in semifinite von Neumann algebras, Math. Z. 195 (1987), 17-27.

21. __ Distance between unitary orbits in von Neumann algebras, Pacific J. Math. 138 (1989), 259-294.

22. C. J. Himmelberg, Measurable relations, Fund. Math. 87 (1975), 53-72.

23. E. Kamei, Double stochasticity in finite factors, Math. Japon. 29 (1984), 903-907.

24. __ An order on statistical operators implicitly introduced by von Neumann, Math. Japon. 30 (1985), 891-895.

25. H. Kosaki, Applications of uniform convexity of noncommutative $L^{p}$-spaces, Trans. Amer. Math. Soc. 283 (1984), 265-282.

26. A. S. Markus, The eigen- and singular values of the sum and product of linear operators, Russian Math. Surveys 19 (1964), 91-120.

27. A. W. Marshall and I. Olkin, Inequalities: Theory of majorization and its applications, Academic Press, 1979.

28. Y. Nakamura, An inequality for generalized s-numbers, Integral Equations Operator Theory 10 (1987), 140-145.

29. ___, $L^{p}$-distance between unitary orbits in type III factors, Preprint.

30. E. Nelson, Notes on non-commutative integration, J. Funct. Anal. 15 (1974), 103-116. 
31. T. Ogasawara and K. Yoshinaga, A non-commutative theory of integration for operators, J. Sci. Hiroshima Univ. 17 (1955), 311-347.

32. V. I. Ovchinnikov, s-numbers of measurable operators, Functional Anal. Appl. 4 (1970), 236-242.

33. D. Petz, Spectral scale of self-adjoint operators and trace inequalities, J. Math. Anal. Appl. 109 (1985), 74-82.

34. J. R. Ringrose, On the Dixmier approximation theorem, Proc. London Math. Soc. (3) 49 (1984), 37-57.

35. Y. Sakai, Weak spectral order of Hardy, Littlewood and Pólya, J. Math. Anal. Appl. 108 (1985), 31-46.

36. I. Segal, A non-commutative extension of abstract integration, Ann. of Math. (2) 57 (1953), 401-457.

37. S. Strătilă and L. Zsidó, An algebraic reduction theory for $W^{*}$-algebras. II, Rev. Roumaine Math. Pures Appl. 18 (1973), 407-460.

38. M. Takesaki, Theory of operator algebras I, Springer-Verlag, 1979.

39. M. Terp, $L^{p}$-spaces associated with von Neumann algebras, Notes, Copenhagen Univ., 1981.

40. R. C. Thompson, Singular values, diagonal elements, and convexity, SIAM J. Appl. Math. 32 (1977), 39-63.

41. H. Umegaki, Conditional expectation in an operator algebra. I, II, III, IV, Tôhoku Math. J. 6 (1954), 177-181; ibid. 8 (1956), 86-100; Kōdai Math. Sem. Rep. 11 (1959), 51-64; ibid. 14 (1962), 59-85.

42. F. J. Yeadon, Non-commutative $L^{p}$-spaces, Math. Proc. Cambridge Philos. Soc. 77 (1975), 91-102.

Division of Applied Mathematics, Research Institute of Applied Electricity, HokKAIDO UNIVERSITY, SAPPORO 060, JAPAN 